\section{An EDS1 EP-domain surface mediating timely transcriptional reprogramming of immunity genes}

3 Deepak D. Bhandari ${ }^{1}$, Dmitry Lapin ${ }^{1}$, Barbara Kracher $^{1}$, Patrick von Born ${ }^{1}$, Jaqueline Bautor ${ }^{1}$,

$4 \quad$ Karsten Niefind ${ }^{2}$, Jane. E. Parker ${ }^{{ }^{*}}$

5

$6 \quad{ }^{1}$ Department of Plant-Microbe Interactions, Max-Planck Institute for Plant Breeding

7 Research, Carl-von-Linne-Weg 10, 50829 Cologne, Germany

$8 \quad{ }^{2}$ Department of Chemistry, Institute of Biochemistry, University of Cologne, Zuelpicher

9 Strasse 47, Cologne, Germany

10 *correspondence: parker@mpipz.mpg.de

11

12 Running Title: Nuclear signalling in plant immunity

13

14

Character count - 36039

15

16

Key words: Arabidopsis, effector-triggered immunity, salicylic acid, coronatine, NLR

17 receptors, defence gene expression 


\section{Abstract}

21 Plant intracellular NLR receptors recognize pathogen interference to trigger immunity. NLR signalling mechanisms have not been resolved. Enhanced disease susceptibility1 (EDS1) heterodimers are recruited by Toll-interleukin1-receptor domain NLRs (TNLs) to transcriptionally mobilize resistance pathways. Using an Arabidopsis EDS1 heterodimer crystal structure we interrogate the conserved but functionally uncharacterized EDS1 a-helical EP-domain. We identify EP-domain positively charged residues lining a cavity that are essential for TNL immunity signalling, beyond heterodimer formation. Mutating arginine (R493) to alanine creates a weak EDS1 allele which disables TNL immunity against bacteria producing a virulence factor, coronatine (COR). Arabidopsis plants expressing EDS1 ${ }^{\mathrm{R} 493 \mathrm{~A}}$ are slow to mobilize defence gene expression changes, independently of COR. The transcriptional delay has severe consequences for pathogen resistance and for countering bacterial COR. We uncover a set of host immunity genes whose repression by COR is blocked by wild-type EDS1 but not by $\mathrm{EDS} 1^{\mathrm{R} 493 \mathrm{~A}}$ in the TNL response. These data uncover an EDS1 signalling surface lining the heterodimer EP-domain cavity which confers timely transcriptional reprogramming of host defence pathways and blocks bacterial virulence in NLR receptor immunity. 


\section{Introduction}

In plants and animals, innate immunity is governed by surface and intracellular receptors. Mammalian innate immune responses provide an initial barrier against microbial infection, and specific pathogen resistance is normally taken over by the adaptive immune system. By contrast, plants depend entirely on panels of germ line-encoded receptors (Jacob et al, 2013; Jones et al, 2016). Plant recognition of specific pathogen virulence components (known as pathogen effectors) is conferred by intracellular nucleotide-binding/leucine-rich repeat (NLR) receptors in a process called effector-triggered immunity (ETI). NLRs directly or indirectly intercept effectors delivered by pathogens to host cells (Cui et al, 2015; Jones et al, 2016; Zhang et al, 2017). NLR-effector recognition causes receptor conformational activation via an ADP/ATP-dependent switch mechanism, which leads to induction anti-microbial defence pathways, often accompanied by localized host cell death at infection sites (the hypersensitive response, HR).

Two major plant NLR classes are defined principally by their N-terminal domain architectures: those with an N-terminal coiled-coil (CC) domain are known as CNLs (or CC-NLRs) and those with a Toll-interleukin 1 receptor (TIR) domain, as TNLs (or TIR-NLRs) (Jacob et al. 2013; Zhang et al. 2017). A characteristic feature of ETI mediated by the different NLR types is amplification of a similar suite of defence pathways that are mobilized at a lower level by surface pattern-recognition receptors (PRRs) recognizing microbe-associated molecular patterns (MAMPs) in basal immunity (Tao et al, 2003; Bartsch et al, 2006; Navarro et al, 2004; Tsuda et al, 2009; Mine et al, 2018; Jacob et al, 2018). Basal immunity pathways are often targeted by pathogen effectors and the transcriptional reestablishment and bolstering of immunity outputs in ETI is a major driver of resistance (Cui et al, 2015; Tsuda \& Somssich, 2015). Current evidence suggests that ETI transcriptional reprogramming is facilitated by the early removal of repression of immunity-related components, setting up waves of transcription factor (TF)-orchestrated changes in host cells and tissues (Cui et al, 2015; Sun et al, 2015; Jacob et al, 2018; Zhou et al, 2018). Numerous TFs have been found to contribute to ETI governed by CNL and TNL receptors(Cui et al, 2015; Birkenbihl et al, 2017). Also, several NLRs have nuclear functions (García \& Parker, 2009; Inoue et al, 2013; Sarris et al, 2015; Le Roux et al, 2015; Fenyk et al, 2015; Cui et al, 2015), suggesting that the path between NLR activation and gene expression reprogramming might in some cases be short. However, the mechanisms by which NLRs modulate transcriptional defences are not known. 
Another emerging NLR ETI characteristic which distinguishes it from generally weaker basal immune responses, is the contribution of alternative (parallel) transcriptional branches, enabling the plant to compensate for disabling of a particular host resistance sector (Tsuda $e t$ al, 2009; Kim et al, 2014b; Cui et al, 2017; Cui et al, 2018). ETI buffering of pathways appears to provide robustness against pathogen interference. For example, Arabidopsis ETI conferred by the TNL pair (RRS1S RPS4) and a CNL (RPS2) receptor recognizing specific effectors delivered by leaf-infecting Pseudomonas syringae pv tomato (Pst) bacteria, engage a prolonged MAP kinase (MPK3/MPK6) cascade and the nucleocytoplasmic, lipase-like regulator Enhanced Disease Susceptibility1 (EDS1) to protect salicylic acid (SA) responsive gene expression outputs (Tsuda et al, 2013; Cui et al, 2017). SA is a phenolic hormone produced by the pathogen-induced enzyme, isochorismate synthase1 (ICS1) (Wildermuth et $a l, 2001$ ), and a central component of plant local and systemic immunity against biotrophic pathogens (Fu \& Dong 2013). SA accumulation is controlled by an ensemble of TFs operating within a phytohormone network, which enables the plant to prioritize responses to a prevailing stress (Seyfferth \& Tsuda, 2014; Pieterse et al, 2012).

Different effectors delivered to host cells by fungal, oomycete and bacterial pathogens target SA immunity, often by boosting the SA-antagonising jasmonic acid (JA) hormone system which mediates resistance to necrotrophic pathogens and insects (Kazan \& Lyons, 2014; Yang et al, 2017). Coronatine (COR) is a potent SA-antagonizing virulence molecule produced by Pseudomonas bacteria (including Pst) which promotes infection by mimicking plant endogenous bioactive JA-isoleucine (JA-Ile) (Brooks et al, 2005; Zheng et al, 2012). Like JAIle, COR signals by binding to nuclear F-box protein coronatine-insensitive1 (COI1) jasmonate ZIM-domain (JAZ) coreceptors, which relieves JAZ repression of a basic helixloop-helix (bHLH) TF, myelocytomatosis oncogene homolog2 (MYC2) (Kazan \& Manners, 2013; Zhang et al, 2015b). MYC2 is a hub TF for JA, ethylene and abscisic acid signalling, orchestrating numerous stress outputs, including transcriptional dampening of SA accumulation (Kazan \& Manners, 2013; Zhang et al, 2015b).

In Arabidopsis and other dicotyledenous plant species, EDS1 is essential for TNL ETI and autoimmune outputs - transcriptional reprogramming, SA accumulation, host cell death and inhibition of pathogen growth (Li et al, 2001; Wiermer et al, 2005; García \& Parker, 2009; Gao et al, 2014; Stuttmann et al, 2016). Hence, EDS1 is an early convergence point for activated TNLs recognizing different pathogens, and a crucial link to downstream pathways. A small nuclear EDS1 pool is sufficient for Arabidopsis basal immunity against virulent 
102

103

104

105

106

107

108

109

110

111

112

113

114

115

116

117

118

119

120

121

122

123

124

125

126

127

128

129

130

131

pathogens and TNL ETI, while cytoplasmic EDS1 contributes to pathogen-triggered host cell death (García et al, 2010; Rietz et al, 2011; Heidrich et al, 2011; Stuttmann et al, 2016).

To signal in TNL ETI, Arabidopsis EDS1 forms separate heterodimer complexes with each of its sequence-related partners, phytoalexin deficient4 (PAD4) and senescence-associated gene101 (SAG101) (Zhou et al, 1998; Jirage et al, 1999; Feys et al, 2001; Feys et al, 2005; Rietz et al, 2011; Wagner et al, 2013). Analysis of the crystal structure of an EDS1-SAG101 heterodimer, and a structural homology-based model of EDS1-PAD4, showed that the partner N-terminal lipase-like ( $a / \beta$-hydrolase fold) domains act as a noncatalytic scaffold for interaction and promoting contacts between the partner C-terminal 'EP' (from EDS1-PAD4) a-helical bundle domains (Rietz et al, 2011; Wagner et al, 2013). The heterodimer EP-domains create a cavity. The function of the EP-domains and associated cavity has not been established.

Arabidopsis EDS1 and SA signalling pathways operate as genetically parallel, mutually reinforcing resistance sectors (Tsuda et al, 2009; Venugopal et al, 2009; Cui et al, 2018). We recently reported that EDS1-PAD4 heterodimers, besides promoting ICS1 expression and SA accumulation, antagonize COR stimulated MYC2 pathways in Arabidopsis TNL ETI against Pst bacteria (Cui et al, 2018). We established that EDS1-PAD4 antagonism of the COR/JA MYC2-branch is independent of its promotion of the ICS1/SA-branch, leading us to propose a two-pronged EDS1 signalling mechanism in ETI for buffering SA immunity against genetic or pathogen interference (Cui et al, 2018).

In this study, we investigate the role of the EDS1 EP-domain in Arabidopsis TNL ETI. By characterizing molecular, transcriptional and disease resistance phenotypes of plants expressing EDS1 structure-guided EP-domain mutations, we identify a positive surface lining the EP-domain heterodimer cavity which is necessary for pathogen resistance and for timely transcriptional reprogramming of immunity genes. Strikingly, the EDS1 EP-domain surface also signals in ETI conferred by a CNL receptor (RPS2), and in both TNL (RRS1 RPS4) and CNL (RPS2) ETI to Pst bacteria, EDS1 EP-domain functions can be dissected into three distinct resistance branches.

\section{Results}

Residues lining the EDS1 heterodimer cavity mediate immunity signalling 
The Arabidopsis EDS1-SAG101 heterodimer crystal structure reveals a cavity formed by the partner EP-domains with several conserved positively charged amino acids (lysines and arginines) on the EDS1 and SAG101 surfaces (Fig. S1A, B) (Wagner et al, 2013). A similar distribution of positive residues was found in a homology-based structural model of the EDS1PAD4 heterodimer (Wagner et al, 2013). We selected solvent accessible EDS1 lysine (K) and arginine (R) residues that are not part of the heterodimer interface and mutated these individually to alanines (Fig. S1A, C). In a yeast 2-hybrid (Y2H) assay, the EDS1 EP-domain amino acid exchanges did not reduce EDS1-PAD4 interaction compared to the EDS1 1 LIF lipase-like domain mutant which fails to bind PAD4 (Fig. S1D) (Wagner et al, 2013). Constructs of wild-type EDS1 cDNA (cEDS1) and EDS1 EP-domain variants under the EDS1 native promoter and N-terminally tagged with yellow fluorescent protein (YFP) were transformed into the Arabidopsis Col eds1-2 null mutant (Wagner et al, 2013; Bartsch et al, 2006). Primary $\left(T_{1}\right)$ transformants for each construct were then inoculated with the oomycete pathogen Hyaloperonospora arabidopsidis ( $\mathrm{Hpa}$ ) isolate EMWA1 to test for TNL (RPP4) immunity phenotypes, measured against resistant and susceptible controls (Stuttmann et al, 2011). Several EP-domain cavity mutants had reduced RPP4 resistance (Fig. S1C). One mutant, EDS1 $1^{\mathrm{R} 493 \mathrm{~A}}$, located in the centre of the cavity (Fig. 1A) was chosen for in-depth analysis because it displayed similarly high Hpa EMWA1 disease susceptibility as eds 1-2. Consistent with the $\mathrm{T}_{1}$ TNL immunity phenotypes, two independent homozygous cEDS1 ${ }^{\mathrm{R} 493 \mathrm{~A}}$ transgenic lines (denoted R493A\#1 and \#2) were fully susceptible to Hpa isolate CALA2 (Fig 1B), recognized in Col by $R P P 2 A$ and $R P P 2 B T N L$ genes (Sinapidou et al, 2004), and the bacterial pathogen Pst AvrRps4 recognized by the nuclear TNL pair RRS1S-RPS4 (Wirthmueller et al, 2007; Williams et al, 2014; Saucet et al, 2015) (Fig. 1C). These data show that EDS1 $1^{\mathrm{R} 493 \mathrm{~A}}$ compromises immunity governed by TNLs recognizing an oomycete and bacterial pathogen, without breaking EDS1 heterodimer formation.

Further examination of the EDS1-SAG101 and EDS1-PAD4 structures revealed a SAG101 and PAD4 EP-domain surface resembling the EDS $1^{\mathrm{R} 493 \mathrm{~A}}$ patch but facing away from the cavity (Fig. 1D). We mutated PAD4 arginine 420 (PAD4 ${ }^{\mathrm{R} 420 \mathrm{~A}}$ ) which is predicted to form similar interactions with neighbouring residues as $\operatorname{EDS}^{\mathrm{R} 493}$ (Fig. 1D), but on the external PAD4 surface. These amino acids are also aligned at the sequence level between EDS1 and PAD4. Wild-type cPAD4 and PAD4 ${ }^{\mathrm{R} 420 \mathrm{~A}}$ controlled by the PAD4 native promoter and N-terminally tagged with YFP were transformed into Col pad4-1 sag101-3 mutant in which loss of SAG101 is compensated for by PAD4 (Feys et al, 2005; Wagner et al, 2013). In RPP4 (Hpa EMWA1) 
infection assays of $\mathrm{T}_{1}$ transgenic lines, PAD4 R420A was as resistant as cPAD4 and Col, whereas the EDS1 R493A\#1, eds1-2 and pad4-1 sag101-3 plants were susceptible (Fig. 1E). These data suggest that the location of positively charged residues, such as EDS1 ${ }^{\mathrm{R} 493}$, within the EP-domain cavity is crucial for TNL immunity.

We tested whether EDS1 $1^{\mathrm{R} 493 \mathrm{~A}}$ retains interaction with PAD4 in planta by performing transient expression and immunoprecipitation (IP) assays in eds1-2 pad4-1 sag 101-3 protoplasts (Cui et $a l, 2018)$. EDS1 ${ }^{\text {R493A }}$ fused to a FLAG tag interacted with PAD4-YFP as strongly as wild-type

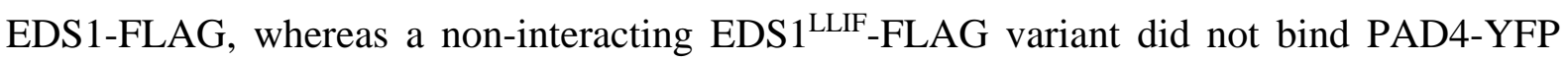
(Fig. S2A). YFP-tagged cEDS1 ${ }^{\text {R493A }}$ protein in the R493A\#1 and R493A\#2 transgenic lines had a similar nucleocytoplasmic distribution as YFP-cEDS1 at $24 \mathrm{~h}$ post infection (hpi) with Pst AvrRps4 (Fig. S2B), indicating that loss of TNL immunity is not due to failed EDS1 nuclear accumulation (García et al, 2010; Stuttmann et al, 2016). However, confocal microscopy imaging of multiple samples showed that YFP-cEDS1 ${ }^{\text {R493A }}$ nucleocytoplasmic fluorescence was lower than YFP-cEDS1 (Fig. S2B). On a protein blot probed with a-GFP antibodies,

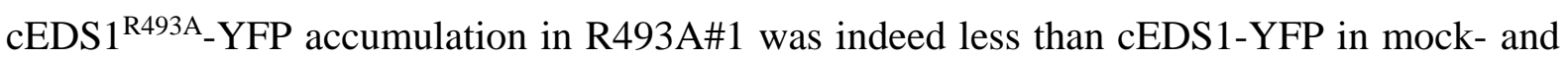
Pst AvrRps4-inoculated leaf extracts (Fig. S2D). To test whether the EDS1 ${ }^{\text {R493A }}{ }^{\text {defect in TNL }}$ immunity is due to its low accumulation, we generated transgenic eds 1-2 lines expressing

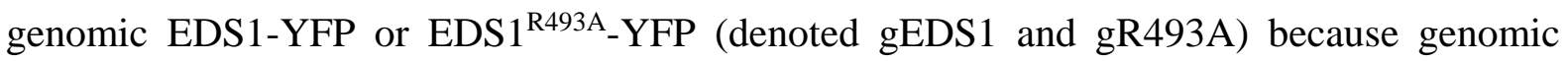
EDS1 is generally more highly expressed than cEDS1 (Stuttmann et al, 2016). Although gEDS1 and gR493A accumulated to higher levels than cEDS1 in mock-treated tissues and to similar levels after Pst AvrRps4 infection (Fig. S2D), the gR493A transgenic line was as susceptible to Pst AvrRps4 as R493A\#1 or eds1-2, compared to Col, gEDS1 and cEDS1 plants (Fig. S2C). These data show that impaired TNL (RRS1S-RPS4) resistance in R493A is not a consequence of low protein accumulation. We concluded that EDS1 ${ }^{\mathrm{R} 493}$ lining the EP-domain cavity confers an important signalling property on the EDS1-PAD4 heterodimer.

\section{EDS1 $^{\text {R493A }}$ delays TNL transcriptional reprogramming}

TNL/EDS1 bacterial immunity divides into two mutually reinforcing resistance branches: one involving SA synthesized by EDS1-induced ICS1, the other working independently of SA and involving EDS1 antagonism of MYC2 (Cui et al, 2017; Cui et al, 2018). To check if SA accumulation is affected in R493A, we measured free (active) SA accumulation in leaves of Col, eds1-2 and cEDS1 and R493A plants at 0, 8 and 24 hpi with Pst AvrRps4. At 8 hpi, SA 
levels in R493A lines \#1 and \#2 remained low, resembling the eds1-2 null mutant (Fig. 2A). At 24 hpi, the R493A lines but not eds 1-2 recovered SA accumulation to similar levels as cEDS1 and Col (Fig. 2A). Low SA accumulation at 8 hpi correlated with reduced expression of the SA-marker gene PRI (pathogenesis related 1) in R493A compared to cEDS1 and Col at $24 \mathrm{hpi}$ (Fig. 2B). These data indicate that $\mathrm{EDS} 1^{\mathrm{R} 493 \mathrm{~A}}$ is not a complete loss-of-function mutation but instead slow to mobilize the SA branch of TNL immunity. Therefore, one function of the EDS1 EP-domain, which is compromised in R493A, is to promote the SA immunity branch.

EDS1 SA-independent signalling operating in parallel with the EDS1-promoted SA/ICS1 branch enables the plant to buffer against a disabled SA sector in ETI (Tsuda et al, 2009; Venugopal et al, 2009; Cui et al, 2018). We performed RNA-seq to interrogate the EDS1 ${ }^{\text {R493A }}$ defect in TNL (RRSIS RPS4) transcriptional reprogramming. Four-week-old Col, eds1-2, cEDS1 and R493A (line \#1) plants were infiltrated with Pst AvrRps4 and three independent biological replicates for each line were processed and analysed at 0, 8 and 24 hpi (see Methods). In a 2-D scatter plot, at 8 hpi there were only 12 DEGs between R493A and eds1-2 (Fig. 2C (pink boxes) and Table S1). These included EDS1 $\left(\log _{2}\right.$ FC 6.03, FDR=9.92E-03) and PBS3 $\left(\log _{2}\right.$ FC 3.7, FDR=2.65E-03). Therefore, at the level of TNL-triggered SA accumulation and transcriptional reprogramming, R493A behaves like the eds $1-2$ null mutant at 8 hpi (Fig. $2 \mathrm{~A}$, C). At 24 hpi, R493A had a markedly different expression profile to eds 1-2 (Fig. 2C) with 2053 DEG $\left(\left|\log _{2} \mathrm{FC}\right| \geq 1, \mathrm{FDR}<0.05\right)$. R493A did not fully recover at 24 hpi as there were 576 DEG $\left(\left(\left|\log _{2} \mathrm{FC}\right| \geq 1, \mathrm{FDR}<0.05\right)\right.$ between R493A and cEDS1 compared with $5993 \mathrm{DEG}\left(\left(\left|\log _{2} \mathrm{FC}\right| \geq 1\right.\right.$, FDR $<0.05$ ) between $e d s 1-2$ and cEDS1 (Fig. 2C). These gene expression profiles show that $\mathrm{EDS1}^{\mathrm{R} 493 \mathrm{~A}}$ fails to mobilize TNL transcriptional reprogramming at 8 hpi but recovers largely at 24 hpi. Together with the disease susceptibility phenotypes of R493A lines \#1 and \#2 (Fig. $1 \mathrm{~B}, \mathrm{C})$, we concluded that recovery of gene expression in R493A at $24 \mathrm{hpi}$ is too late to halt pathogen growth. Hence, integrity of the EDS1 EP-domain appears to be critical for timely TNL-triggered transcriptional defence reprogramming.

\section{EDS1 $^{\text {R493A }}$ fails to antagonize bacterial COR stimulated MYC2 in ETI}

Pst DC3000 produces the JA-Isoleucine (JA-Ile) mimic coronatine (COR) to promote JA response pathways and suppress SA signalling (Brooks et al, 2005; Zheng et al, 2012; Geng et al, 2014). One function of the EDS1-PAD4 heterodimer in RRS1S RPS4 ETI is to inhibit COR stimulation of MYC2-regulated JA pathway genes independently of ICS1 (Cui et al, 2018). 
Because R493A displayed a general delay in gene expression reprogramming (Fig. 2C) and accumulation of SA (Fig. 2A), we tested whether EDS1 ${ }^{\mathrm{R} 493 \mathrm{~A}}$ is defective in antagonizing COR promoted bacterial infection. For this, Pst AvrRps 4 or the COR-deficient Pst $\Delta$ cor AvrRps 4 strain were infiltrated into leaves of Col, eds 1-2, cEDS1 and R493A lines \#1 and \#2, and bacterial growth measured at $3 \mathrm{dpi}$. As expected, Col and cEDS1 were equally resistant to $P s t$ AvrRps4 and Pst $\Delta$ cor AvrRps4, reflecting strong EDS1 antagonism of COR stimulated bacterial growth in RRSIS RPS4 ETI (Fig. 3A). The eds 1-2 mutant was susceptible to both Pst AvrRps4 strains but supported 1.5-log lower Pst $\Delta$ cor AvrRps4 growth compared to Pst AvrRps 4 , indicating both COR stimulated and COR-independent bacterial growth effects in the absence of EDS1 (Fig. 3A) (Cui et al, 2018). Notably, while R493A lines displayed eds1-2 level susceptibility to Pst AvrRps4, they were as resistant as Col and cEDS1 to Pst $\Delta$ cor AvrRps4 (Fig. 3A). Therefore, loss of R493A resistance to Pst AvrRps4 is due to its failure to antagonize bacterial COR, presumably signalling via MYC2. To verify this, we crossed cEDS1 and R493A\#1 into an eds1-2 myc2-3 mutant background. Whereas R493A (in eds1-2) was as susceptible as eds1-2, R493A eds1-2 myc2-3 was as resistant as Col and cEDS1 (Fig. 3B), indicating that $\mathrm{EDS}^{\mathrm{R} 493 \mathrm{~A}}$ recovers resistance to Pst AvrRps4 when $M Y C 2$ is mutated. These data point to a defect of $\mathrm{EDS}^{\mathrm{R} 493 \mathrm{~A}}$ in counteracting COR-dependent, MYC2 promoted bacterial growth in TNL ETI. With respect to Pst AvrRps4, therefore, EDS1 ${ }^{\text {R493A }}$ loss of resistance is conditional on COR stimulation of the MYC2 JA signalling branch.

Because EDS1 ${ }^{\mathrm{R} 493 \mathrm{~A}}$ was also compromised in TNL ( $R P P 4$ and RPP2A, B) immunity to Hpa (Fig. 1B, E), we tested whether there is a recovery of $R P P 4$ resistance to Hpa EMWA1 in the eds1-2 myc2-3 transgenic lines. Col and myc2-3 expressed full RPP4 resistance after quantifying EMWA1 sporulation on leaves (Fig. 3C). There were similar high levels of EMWA1 sporulation in eds1-2, eds 1-2 myc2-3, R493A eds1-2 and R493A eds1-2 myc2-3 (Fig. 3C). These data show that increased susceptibility to Hpa EMWA1 in R493A is not dependent on $M Y C 2$. Therefore, the EDS1 EP-domain and associated heterodimer cavity have broader functions in TNL immunity than antagonizing MYC2.

\section{EDS1 $^{\text {R493A }}$ TNL signalling delay is compounded by bacterial COR}

Because COR-activated MYC2 represses SA accumulation (Brooks et al, 2005; Zheng et al, 2012; Cui et al, 2018) and $\mathrm{EDS} 1^{\mathrm{R} 493 \mathrm{~A}}$ causes delay in SA accumulation at 8 hpi with $P s t$ AvrRps4 (Fig. 2A), we tested whether resistance of R493A to Pst $\Delta$ cor AvrRps4 (Fig. 3A) is 
due to restored SA. Col and cEDS1 accumulated similar levels of free SA at 8 hpi with Pst AvrRps4 and higher SA in response to Pst $\Delta$ cor AvrRps4, consistent with COR dampening of SA accumulation (Fig. 4A). The eds1-2 null mutant failed to accumulate SA in response to either strain (Fig. 4A), fitting with EDS1 promotion of ICS1 expression and SA independently of its suppression of MYC2 in TNL (RRS1S RPS4) immunity (Cui et al, 2018). Strikingly, R493A lines \#1 and \#2 failed to accumulate SA in response to Pst AvrRps4 and Pst $\Delta$ cor AvrRps4 (Fig. 4A). Therefore, delayed SA accumulation in R493A is not caused by its failure to antagonize bacterial COR. These data suggest that antagonism of COR/MYC2 signalling and promotion of SA accumulation are distinct properties of the EDS1-PAD4 heterodimer EPdomain, and more precisely EDS1 ${ }^{\mathrm{R} 493}$ lining the EP cavity, in TNL ETI.

Next, we examined which gene expression sectors affected by bacterial COR might explain the compromised TNL ETI in R493A, by performing an RNA-seq analysis at 8 and 24 hpi with Pst $\Delta$ cor AvrRps4. To compare between Pst AvrRps4 and Pst $\Delta$ cor AvrRps4 RNA-seq experiments, gene expression data for all lines was normalized against the respective Col control within each treatment (see Methods). A multi-dimensional scaling (MDS) plot shows that cEDS1 and Col expression profiles clustered together at 8 and 24 hpi for both treatments (Fig. 4B). At 8hpi, R493A clustered away from cEDS1 and eds1-2 with Pst $\Delta$ cor AvrRps 4 but close to eds1-2 with Pst AvrRps4 (Fig. 4B, coloured circles). At 24hpi, the R493A transcriptome was similar to cEDS1 with Pst $\Delta$ cor AvrRps4 (filled triangles) but distinct from cEDS1 with Pst AvrRps4 (filled squares) (Fig. 4B). This analysis shows that EDS1 ${ }^{\text {R493A }}$ causes a general delay in TNL gene expression reprogramming which is exacerbated by bacterial COR. The trend fits with the SA accumulation profiles (Fig. 4A) and emphasizes the importance of EDS1 EP-domain for rapid transcriptional mobilization of multiple pathways besides blocking bacterial COR actions in TNL ETI. We compared our transcriptome data at 8 hpi with publicly available SA- and JA-responsive transcriptomes (Tsuda et al, 2009; Hickman et al, 2017). This showed that R493A exhibits lower expression of genes regulated by ICS1 compared to cEDS1 regardless of bacterial COR status (Fig. S3A). In contrast, genes that are repressed by JA were also reduced in R493A infiltrated with Pst AvrRps4 but not with Pst $\Delta c o r$ AvrRps4 (Fig. S3A). Thus, the R493A transcriptome reflects both slow mobilisation of SA pathways and a defect in antagonising COR/MYC2-regulated JA pathways.

We next searched for DEG between Pst AvrRps4 and Pst $\Delta$ cor AvrRps4 treatments in the different lines at 8 and 24 hpi. After hierarchical clustering, a derived expression heatmap revealed the extent of delay in R493A at 8 hpi compared to cEDS1, both in the presence and 
absence of bacterial COR (Fig. 4C). Only 75 (8 hpi) and 3 (24 hpi) DEG spread across different clusters were found for cEDS1, reinforcing the notion that EDS1 effectively antagonizes COR in TNL ETI. There were major expression differences between Pst AvrRps4 and Pst $\Delta$ cor AvrRps 4 treatments (2810 at 8 hpi and 987 at 24 hpi) in the eds 1-2 null mutant, consistent with EDS1 loss-of-function failing to counter COR effects. While R493A was slow in ETI transcriptional reprogramming against both Pst AvrRps4 and Pst $\Delta$ cor AvrRps4 (Fig. 4B), there remained 1009 (8 hpi) and 210 (24 hpi) DEG ( $\log 2 \mathrm{FC} \mid \geq 1, \mathrm{FDR} \leq 0.05)$ between these treatments in R493A (Table S2), indicating that the transcriptional delay in R493A is compounded by COR. The 210 DEG found in R493A at 24 hpi mostly overlapped with DEG in $e d s 1-2$. These expression changes are therefore probably not important for TNL immunity to Pst AvrRps4. Because R493A is susceptible to Pst AvrRps4 but resistant to Pst $\Delta$ cor AvrRps4 infection (Fig. 3A), we reasoned that EDS1 interference with COR-dependent expression changes before or at $8 \mathrm{hpi}$ is crucial for halting bacterial growth in the TNL immune response.

\section{COR represses a distinct set of immunity-related genes in $\mathbf{R} 493 \mathrm{~A}$}

One expression cluster (\#17) stood out in the above analysis because it contains EDS1dependent DEG at 8 hpi that are more highly expressed in R493A with Pst $\Delta$ cor AvrRps 4 compared to Pst AvrRps4 (Fig. 4C, Table S3), suggesting there is targeted repression of a set of genes by COR in R493A but not in Col or cEDS1 lines (which effectively counter COR effects) in TNL immunity. Cluster \#17 comprises 383 genes associated with Gene Ontology (GO) terms phosphorylation (37/376 eg. MPK3, MPK2), cell death (18/376 eg. RPS2, NPR1, CPR5) and defence response (73/376 eg. RPS4, RPP4, ADR1-L2) (Table S4). Notable members of cluster \#17 are functionally defined NLR (TNL and CNL) and WRKY family TF genes (Fig. 4C, Table S3).

We found a significant overlap between cluster \#17 genes and genes regulated by the SA analogue BTH (Yang et al, 2017) or JA (Hickman et al, 2017). Here, cluster \#17 genes fall into four categories (Fig. 4D; Table S5), the largest of which (i) has genes induced by BTH and not repressed by JA (169/383), followed by (ii) genes induced by BTH and repressed by JA (113/383), (iii) genes not responsive to either BTH or JA (82/383), and a small group (iv) genes that are repressed by JA only (19/383). This comparison suggests that TNL/EDS1 signalling at 8 hpi protects a large number of SA/JA responsive but also SA/JA-unrelated immunity genes from bacterial COR repression. Only 30\% (118/383) cluster \#17 genes overlap with a set of 
ETI-associated genes that were extracted from transcriptomic analyses of the Arabidopsis RPS2 (CNL) response to AvrRpt2 expressed in planta or delivered by Pst bacteria (Hatsugai et al, 2017; Mine et al, 2018) (Fig. S3B). Therefore, the cluster \#17 represents a new and potentially interesting ETI-related gene set. We did not detect significant enrichment of particular cis-regulatory elements in the promoters of cluster \#17 genes (by MEME, http://meme-suite.org), suggesting that these genes are controlled by multiple TFs.

332

In summary, comparison of cEDS1, eds1-2 and R493A transcriptomes between Pst AvrRps4 and Pst $\Delta$ cor AvrRps 4 treatments uncovered a set of TNL/EDS1-controlled genes (cluster \#17) whose repression in response to bacterial COR at $8 \mathrm{hpi}$ is inadequately blocked by EDS1 ${ }^{\mathrm{R} 493 \mathrm{~A}}$. Hence, R493A reveals a function of the EDS1 heterodimer EP-domain in countering bacterial COR repression of host immunity genes within the first $8 \mathrm{~h}$ of TNL ETI.

\section{A positive charge at $\mathrm{EDS1}^{\mathrm{R} 493}$ is essential for TNL immunity}

We have shown that mutations of positively charged residues in the EDS1 EP cavity impaired EDS1 TNL functions (Fig. S1C, 1B, 1C). To test the importance of the charge at R493, we generated positively (lysine, $\mathrm{EDS}^{\mathrm{R} 493 \mathrm{~K}}$ ) and negatively (glutamate, EDS1 ${ }^{\mathrm{R} 493 \mathrm{E}}$ ) charged variants of EDS1 ${ }^{\mathrm{R} 493}$. Like EDS1 ${ }^{\mathrm{R} 493 \mathrm{~A}}$, YFP-tagged EDS1 ${ }^{\mathrm{R} 493 \mathrm{~K}}$ and EDS1 ${ }^{\mathrm{R} 493 \mathrm{E}}$ displayed wildtype nucleocytoplasmic localization in transient expression assays (Fig. S4A). The FLAGtagged EDS1 ${ }^{\text {R493 }}$ variants interacted with PAD4-YFP in IP experiments (Fig. S4B), consistent with the charge at the EP cavity not affecting heterodimer formation. Additionally, none of the FLAG-tagged EDS1 ${ }^{\mathrm{R} 493}$ variants altered interactions between PAD4-YFP and StrepII-HA (SH)-tagged MYC2 in IPs of transiently expressed proteins (Fig. S4C), indicating that an altered EDS1 ${ }^{\mathrm{R} 493}$ charge does not disturb EDS1-PAD4 association with MYC2.

Two independent homozygous $\mathrm{EDS}^{\mathrm{R} 493 \mathrm{~K}}$ and $\mathrm{EDS} 1^{\mathrm{R} 493 \mathrm{E}}$ transgenic lines (respectively, R493K and R493E\#1 and \#2) in eds1-2 were tested for TNL (RPP4) resistance to Hpa EMWA1. R493E was as susceptible as eds1-2 and R493A, whereas R493K expressed full $R P P 4$ resistance, as monitored by trypan blue staining of infected leaves (Fig. 5A). Therefore, a positive charge at EDS1 amino acid 493 rather than an arginine per se is required for TNL immunity to Hpa EMWA1. 
AvrRps4. Measured against Col, cEDS1 and eds1-2, R493K was fully resistant while R493E was fully susceptible to both Pst AvrRps4 strains, whereas R493A was susceptible only to Pst AvrRps4 (Fig. 5B). Free SA accumulation in the R493K and R493E lines at 8 and 24 hpi with Pst AvrRps4 or Pst $\Delta$ cor AvrRps4 mirrored, respectively, cEDS1 and eds1-2 (Fig. 5C). The same trend was found at the level of expression of marker genes (EDS1, PAD4 and ICS1) for the EDS1/PAD4-induced SA immunity branch at 8 hpi (Fig. S5A). These SA accumulation, gene expression and disease resistance phenotypes show that R493K phenocopies wild-type and R493E the eds1-2 null mutant. We performed qRT-PCR analysis of MYC2-branch JA response marker genes SA methyl transferase 1 (BSMT1), JAZ10 and Vegetative Storage Protein 1 (VSPl) in the R493 variants at 24 hpi with Pst AvrRps4 (Cui et al, 2018). Here, R493K behaved like wild-type cEDS1 and R493E like eds1-2 (Fig. S5B). By contrast, R493A repressed BSMT1, JAZ10 and VSP1 MYC2-branch genes almost as strongly as cEDS1 (or Col) (Fig. S5B). Hence, EDS1 ${ }^{\text {R493A }}$ antagonized the COR-stimulated MYC2-branch at 24 hpi but was unable to counter Pst AvrRps4 infection in ETI (Fig. 3A). Therefore, near wild-type suppression of MYC2-branch genes in R493A plants at 24 hpi was insufficient for TNL ETI. Together, these data show that a positive charge at $\operatorname{EDS}^{\mathrm{R} 493}$ is critical for timely defence gene expression changes and pathogen resistance in TNL ETI.

\section{EDS1 $^{\mathrm{R} 493 A}$ residual signalling function is specific to ETI}

EDS1-PAD4 complexes confer basal immunity to virulent $P$ st DC3000 in the absence of TNLeffector recognition (Jirage et al, 1999; Feys et al, 2001; Rietz et al, 2011; Wagner et al, 2013). We therefore tested whether EDS1 ${ }^{\mathrm{R} 493 \mathrm{~A}}$ restriction of Pst DC3000 growth is conditional on bacterial COR by infiltrating leaves of the different EDS1 $1^{\mathrm{R} 493}$ transgenic lines with $P s t$ DC3000 or Pst $\Delta$ cor. Col, cEDS1 and R493K expressed similar resistance to each bacterial strain, with $\sim 10$-fold higher Pst DC3000 growth than Pst $\Delta$ cor at 3 dpi (Fig. 6A). The eds1-2, R493A and R493E lines were similarly susceptible to each strain, with 100-fold higher Pst DC3000 growth compared to Pst $\Delta$ cor (Fig. 6A). Therefore, R493A does not recover basal resistance to Pst $\Delta c o r$, whereas it recovers TNL (RRS1S RPS4) ETI to Pst $\Delta$ cor AvrRps4 (Fig. 3A, 5B). This result shows that while a positive charge at $\operatorname{EDS} 1^{\mathrm{R} 493}$ is necessary for TNL ETI and basal immunity against $P$ st bacteria, EDS1 ${ }^{\mathrm{R} 493 \mathrm{~A}}$ is partially equipped for TNL ETI but unequipped for basal immunity even without resistance repressive effects of bacterial COR. 
The ICS1/SA pathway can partially buffer eds 1 loss-of-function in TNL (RRS1S RPS4) ETI sector and bacterial COR effects on R493A function in TNL ETI. For this, cEDS1 and R493A transgenic lines were crossed into an eds1 ics1 (eds1-2 sid2-1) mutant background and growth of Pst $\Delta$ cor AvrRps 4 was measured at 3 dpi. As expected, both cEDS1 and R493A (in eds1-2) conferred full TNL immunity to Pst $\Delta$ cor AvrRps4, as did cEDS1 in eds1-2 sid2-1 (Fig. 6B), consistent with EDS1 covering for loss of ICS1-generated SA in TNL ETI (Cui et al, 2017). By contrast, R493A eds1-2 sid2-1 supported intermediate Pst $\Delta$ cor AvrRps4 growth between cEDS1 eds1-2 sid2-1 and eds1-2 sid2-1 (Fig. 6B). Thus, ICS1 generated SA compensates for R493A weak function in RRS1S RPS4 ETI.

The ICS1/SA pathway compensates fully for loss of EDS1 in CNL ETI mediated by two CNL receptors (RPS2 and HRT (HR to turnip crinkle virus)) (Venugopal et al, 2009; Cui et al, 2017). We tested CNL (RPS2) immunity in the above R493A lines by infiltrating leaves with Pst AvrRpt2. In these bacterial growth assays, R493A in eds1-2 sid2-1 also exhibited intermediate susceptibility between cEDS1 eds1-2 sid2-1 and eds1-2 sid2-1 (Fig. 6C). Together, these data show that the same EDS1 EP-domain surface functions in bacterial resistance mediated by a TNL receptor pair and a CNL receptor, and that in both ETI systems the weak resistance signalling activity of EDS1 ${ }^{\mathrm{R} 493 \mathrm{~A}}$ is buffered by ICS1 generated SA. Interestingly, after stripping away SA and COR/JA effects, a portion of EDS1 controlled resistance was detected in R493A eds 1-2 sid2-1 compared to eds 1-2 sid2-1 plants (Fig. 6B). Because the remaining resistance in R493A plants is independent of SA/COR effects in TNL ETI, we refer to it as sector X in a model (Fig.7).

\section{Discussion}

In Arabidopsis, EDS1-PAD4 and EDS1-SAG101 heterodimers, formed principally by the two partner N-terminal lipase-like domains, are required for TNL-triggered ETI and basal immunity against host-adapted bacterial (Pst) and oomycete ( $\mathrm{Hpa}$ ) pathogens (Parker et al, 1996; Feys et al, 2001; Feys et al, 2005; Jirage et al, 1999; Wagner et al, 2013). Current evidence suggests that the partner $N$-terminal lipase-like domains with characteristic $\alpha / \beta$ hydrolase folds serve as a non-catalytic scaffold to stabilize the heterodimer, bringing together the essential a-helical EP-domains to form a cavity (Rietz et al, 2011; Wagner et al, 2013). The 
EP-domains have no known structural homologies outside the EDS1 family (Wagner et al, 2013). Here, using a combination of structure-guided mutants and reductionist genetic approaches, we identify conserved, positively charged residues (K440/441, K478 and R493) lining the EDS1 EP-domain cavity which are crucial for immunity signalling beyond heterodimer formation. By characterizing EDS1 partial- and loss-of-function mutations in the EP-domain, we establish that timely transcriptional mobilization of host immune response pathways is necessary for effective TNL ETI to Pst bacteria and that an important early EPdomain function of EDS1 complexes is to counter COR repression of immunity genes.

We focused our analysis on a single conserved, positively charged EP-domain residue, $\mathrm{EDS}^{\mathrm{R} 493}$, because mutation of this to a neutral alanine (R493A) caused complete loss of TNL immunity to Pst AvrRps4 and two Hpa strains, EMWA1 and CALA2 (Fig. 1B, C, E), indicating its broad importance for TNL ETI. A positive EDS1 ${ }^{\mathrm{R} 493}$ character rather than the arginine per se is required for ETI activity because an R493K exchange behaved as wild-type EDS1, and R493E as the null eds1-2 mutant, in Pst AvrRps4 and Hpa EMWA1 infection assays (Fig. 5A, B). Thus, altering the charge of the amino acid side-chain at position R493 has a strong effect on heterodimer function without disturbing its interfaces (Fig. 5A, B, S4B). Maintained partner interactions of the $\mathrm{EDS}^{\mathrm{R} 493 \mathrm{~A}}, \mathrm{EDS}^{\mathrm{R} 493 \mathrm{~K}}$ and $\mathrm{EDS} 1^{\mathrm{R} 493 \mathrm{E}}$ variants coupled with current molecular modelling suggest that the charge differences are unlikely to destroy integrity of the EP-domain but rather modify ionic interactions at the EP-domain cavity. The cavity surface around $\mathrm{EDS} 1^{\mathrm{R} 493}$ is comprised of several conserved positively charged residues (Fig. S1A). A similar EP-domain surface was identified in PAD4 outside the cavity in which R420 forms comparable interactions with neighbouring glutamic acid and tyrosine residues as EDS1 ${ }^{\mathrm{R} 493}$ (Fig. 1D). However, a PAD4 ${ }^{\text {R420A }}$ exchange did not compromise TNL (RPP4) ETI (Fig. 1E), suggesting that the location and charge of $\mathrm{EDS}^{\mathrm{R} 493}$ within the EP cavity are essential for EDS1-PAD4 function. We anticipate that functional interactions between the heterodimer EPdomains and other proteins or molecules are determined by the charge status of EDS $1^{\mathrm{R} 493}$ and likely other positive residues (Fig. S1C). The JA/COR stimulated master TF MYC2 is not a candidate for direct EP cavity binding because MYC2 likely associates indirectly with EDS1PAD4 complexes (Cui et al, 2018), and their association was not obviously affected by different mutations at $\operatorname{EDS}^{\mathrm{R} 493}$ (Fig. S4C). Also, the partial ability of EDS1 ${ }^{\mathrm{R} 493 \mathrm{~A}}$ to antagonize COR/MYC2 controlled JA-branch genes at 24 hpi in RRS1S RPS4 ETI to Pst AvrRps4 did not correlate with altered EDS1-PAD4 interaction with MYC2 (Fig. S5B). 
One striking feature of $\operatorname{EDS} 1^{\mathrm{R} 493 \mathrm{~A}}$ is that loss of TNL immunity to Pst AvrRps4 is conditional on bacterial COR signalling via MYC2, as indicated by Pst AvrRps4 and Pst Acor AvrRps 4 growth differences in R493A transgenic lines and fully restored resistance to Pst AvrRps4 in an R493A eds1-2 myc2-3 background (Fig. 3A, B). By contrast, EDS1 ${ }^{\text {R493A }}$ behaves as a complete loss-of-function mutation in basal resistance to virulent Pst DC3000 or Pst $\triangle$ cor (Fig. 6A). Thus, TNL (RRSIS RPS4) effector recognition and/or activation (Williams et al, 2014; Sarris et al, 2015; Le Roux et al, 2015) appears to equip EDS1 complexes, via the EP-domain positively charged surface, to block COR/MYC2 stimulated bacterial growth (Fig. 3A, 5B). We reported previously genetic and molecular evidence that TNL/EDS1 interference with COR stimulated MYC2 activity occurs inside nuclei coincident with or after COR is sensed by host COI1-JAZ complexes to release MYC2 from repression (Kazan \& Manners, 2013; Zhang et al, 2015a; An et al, 2017; Cui et al, 2018). An additional TNL (RRS1S RPS4) ETI output might be to lower bacterial COR production during infection because an in planta Pst transcriptomic study showed that Pst COR biosynthesis genes were down-regulated at 6 hpi as part of the RRS1S RPS4 immune response (Nobori et al, 2018).

Importantly, loss of TNL (RPP4) immunity to Hpa EMWA1 in R493A was not recovered by mutation of MYC2 (Fig. 3C). Therefore, EDS1 heterodimers, via the EP-domains, must mobilize other anti-microbial pathways or processes independently of antagonizing COR/MYC2 signalling for TNL immunity against $\mathrm{Hpa}$. It will be interesting to use the plant mutant combinations described here to pare down which pathways are responsible for stopping Hpa growth, building on earlier gene expression microrarray analyses of ETI responses to Hpa isolates (Eulgem et al, 2004; Wang et al, 2011). A number of protein effectors delivered to plant host cells by Hpa, fungal pathogens and P. syringae bacteria suppress SA immunity by targeting JA signalling to shift hormone balance away from SA (Kazan \& Lyons, 2014; Yang et al, 2017). For example, $P$. syringae type III-secreted effectors HopX1 and HopZ1 derepress JA response genes independently of bacterial COR (Jiang et al, 2013; Gimenez-Ibanez et al, 2014). P. syringae effector HopBB1 activates a subset of JA outputs by increasing the binding between two JA response repressors, JAZ3 and TCP14, leading to their COI1-mediated elimination (Yang et al, 2017). Hence, microbes target SA-JA crosstalk in various ways to promote infection. Because EDS1 complexes effectively block bacterial COR virulence in RRS1S RPS4 ETI (Fig. 3A, 5B) and in our RNA-seq data comparisons, we find a significant overlap between TNL/EDS1-dependent DEG and SA/JA-responsive genes (Fig. S3A, 4D), we speculate that one conserved role of EDS1 heterodimers is to protect the plant phytohormone 
network from interference by multiple pathogen effectors at various junctions, in order to preserve SA immunity.

A further insight to TNL/EDS1 ETI gained from our analysis is that perturbation of the EDS1 EP-domain in R493A lines causes an intrinsic delay in transcriptional reprogramming of Arabidopsis defence pathways, independently of bacterial COR. This is seen most clearly at the level of free (active) SA accumulation at 8 hpi and 24 hpi (Fig. 2A, 4A) and in RNA-seq analyses of wild-type Col (or cEDS1), eds1-2 and R493A responses at 8 hpi and 24 hpi with Pst AvrRps4 and Pst $\Delta$ cor AvrRps4 (Fig. 4B, C). Therefore, timing of TNL/EDS1 induction of the ICS1/SA resistance branch and probably numerous other anti-microbial outputs (before or at $8 \mathrm{hpi}$ ) is not dictated by bacterial COR but the plant vulnerable to COR-producing Pst AvrRps4 infection (Fig. 3A, 6B), and possibly also to TNL-recognized Hpa strains (Fig. 1B, E).

Partial recovery of gene expression changes in R493A lines at 24 hpi with Pst AvrRps4 without restoration of RRS1S RPS4 immunity (Fig. 2, 4B, S5B), emphasizes that there is a critical expression time-window for ETI to succeed. Based on these data, we propose that effective TNL/EDS1 immunity involves a crucial early step for rapid transcriptional activation of a broad set of defence pathways, rather than selectively mobilizing resistance outputs. This makes sense because various ETI and basal immune responses differ principally in timing and amplitude of transcriptional reprogramming, while the topology of co-expression networks appears to be quite stable (Tao et al, 2003; Tsuda et al, 2009; Kim et al, 2014; Hatsugai et al, 2017; Mine et al, 2018). Moreover, ETI specifically triggered by one pathogen effector or strain confers broad-spectrum immunity to a range of pathogen types (Rentel et al, 2008).

A recent time-resolved transcriptome study using single and higher order mutants of hormonal pathways indicates broad-sweep transcriptional changes rather than pathway specific signalling cascades in ETI (Mine et al, 2018), stressing the limited value of strong loss-offunction mutations in identifying causal resistance outputs. By comparing the Pst AvrRps 4 and Pst $\Delta$ cor AvrRps4 transcriptomes in a weak EDS1 ${ }^{\mathrm{R} 493 \mathrm{~A}}$ mutant we were able to filter an interesting set of immune-related DEG (cluster \#17) (Fig. 4C, Table S3). These genes would not have emerged from analysis of the cEDS1 and null eds1-2 mutant responses alone because they were repressed at 8 hpi with Pst AvrRps4 only in R493A tissues (Fig. 4C). Cluster \#17 contains a number of functionally defined sensor NLR and WRKY TF genes, and one member (ADR1-L2) of a family of conserved helper NLRs (Fig. 4C) which contribute to ETI (Bonardi 
et al, 2011; Schön et al, 2013; Dong et al, 2016). Only one third of cluster \#17 genes are represented in 'ETI-related' gene sets extracted from RPS2 (CNL)-triggered transcriptomic analyses (Fig. S3B) (Hatsugai et al, 2017; Mine et al, 2018). The two thirds of cluster \#17 'specific' genes might represent another layer of immunity gene expression control in TNL ETI. It is tempting to speculate that susceptibility of R493A to Hpa (Fig. 1B, C) might be due in part to a failure to block $\mathrm{Hpa}$ effectors from targeting some or all of these genes for repression.

We have identified EDS1 EP-domain residues responsible for timely activation of transcriptional reprogramming, beyond heterodimer formation. Analysis of EDS1 ${ }^{\mathrm{R} 493 \mathrm{~A}}$ reinforces a two-pronged EDS1 transcriptional mechanism for RRS1S RPS4 ETI proposed by Cui et al. (2018) which is (i) promotion of ICS1 generated SA and (ii) blocking COR/MYC2 suppression of SA immunity. Delayed free SA accumulation in R493A plants at 8 hpi with Pst AvrRps4 and Pst $\Delta$ cor AvrRps4 (Fig. 4A) highlights a failure of this weak EDS1 allele to rapidly mobilize the SA-branch independently of COR. Early antagonism of COR/MYC2 signalling is important in RRS1S RPS4 ETI because EDS1 $1^{\mathrm{R} 493 \mathrm{~A}}$ is fully susceptible to Pst AvrRps4 despite being able to suppress COR/MYC2 marker genes almost as well as cEDS1 at 24 hpi (Fig. 2C, 4C, S5B). Notably, EDS1 ${ }^{\mathrm{R} 493 \mathrm{~A}}$ retains a portion of RRS1S RPS4 (TNL) resistance to Pst $\triangle$ cor AvrRps4 after removal of ICS1 in an eds1-2 sid2-1 double mutant (Fig. 6B). We therefore add a third yet unexplored EDS1 ETI signalling branch (denoted X in Fig. 7) which is independent of SA and JA/COR crosstalk. By testing R493A eds1-2 sid2-1 plants in ETI conferred by a CNL receptor (RPS2 recognizing Pst AvrRpt2) (Kunkel et al, 1993; Venugopal et al, 2009) (Fig. 6C), it becomes clear that the EDS1 EP-domain confers a crucial function in immunity triggered by TNL and certain CNL receptor types, which is worth exploring further. 
Materials and Methods

\section{Plant materials, growth conditions and pathogen strains}

545

546

547

548

549

550

551

552

553

554

555

556

557

558

559

560

561

562

563

564

565

566

567

568

569

570

571

572

573

574

575

576

577

578

579

580

581

582

583

584

All mutants and lines are in the Col-0 genetic background. The mutants eds 1-2, sid2-1, eds 1-2 sid2-1, myc2-3, pad4-1 sag101-3, eds1-2 myc2-3, as well as YFP-cEDS1 and gEDS1-YFP eds1-2 transgenic lines were previously described (Cui et al, 2018; Wagner et al, 2013; García et al, 2010). Pseudomonas syringae pv. tomato (Pst) strain DC3000, Pst $\Delta$ cor, Pst DC3000 AvrRps4 (Pst AvrRps4), DC3000 Acor AvrRps4 (Pst Dcor AvrRps4) and DC3000 AvrRpt2 (Pst AvrRpt2) are described (Cui et al, 2017). Plants were grown on soil in controlled environment chambers under a $10 \mathrm{~h}$ light regime $\left(150-200 \mu \mathrm{E} / \mathrm{m}^{2} \mathrm{~s}\right)$ at $22{ }^{\circ} \mathrm{C}$ and $60 \%$ relative humidity.

\section{Pathogen infection assays}

For bacterial growth assays, Pst AvrRps4 or Pst $\Delta$ cor AvrRps4 (OD600 $=0.0005)$ in $10 \mathrm{mM}$ $\mathrm{MgCl}_{2}$ were hand-infiltrated into leaves of four-week-old plants and bacterial titers measured at $4 \mathrm{~h}$ post infiltration (day 0) and day 3 as described (Feys et al, 2005). Each biological replicate consists of three leaf disks from different plants and data shown in each experiment is compiled from 3-4 biological replicates. Statistical analysis was performed either using one-way ANOVA with multiple testing correction using Tukey's HSD ( $p<0.005)$.

For gene expression and protein accumulation assays, leaves from four-week-old plants were hand-infiltrated with bacteria $\left(\mathrm{OD}_{600}=0.005\right)$ and samples taken at indicated time points. For measuring protein accumulation, samples were pooled from at least three different plants. For gene expression analysis by qRT-PCR, four or more leaves from different plants were pooled as one biological replicate and two biological replicates were used in each independent experiment. Statistical analysis was performed by Student's t-test with multiple testing correction using Bonferroni method $(\mathrm{p}<0.05)$.

Hpa isolates EMWA1 and CALA2 were sprayed on 2-3 week-old plants at $4 * 10^{4}$ spores $/ \mathrm{ml}$ $\mathrm{dH}_{2} \mathrm{O}$. Plant host cell death and $\mathrm{Hpa}$ infection structures were visualized under a light microscope after staining leaves with lactophenol trypan blue as described (Muskett et al, 2002). $T_{1}$ complementation assays of Arabidopsis transgenic lines were performed as previously described (Stuttmann et al, 2011) and Hpa-infected seedlings rescued by spraying with Ridomil Gold (Syngenta). To quantify Hpa sporulation on leaves, three pots of each genotype were infected and treated as biological replicates. Plants were harvested at 6 dpi, their fresh weight determined, and conidiospores suspended in 5-10 $\mathrm{ml} \mathrm{dH}_{2} \mathrm{O}$ and counted under the microscope using a Neubauer counting chamber.

\section{RNA isolation, library preparation and sequencing}

For RNA-seq experiments, Col, eds1-2, cEDS1 and R493A\#1 four-week-old plants were infiltrated with Pst AvrRps4 or Pst $\Delta$ cor AvrRps4 using the same bacterial titer as for gene expression assays. To randomize samples and reduce variation, total RNA was isolated from four individual plants per genotype (three infected leaves per plant) and pooled as one biological replicate. Each biological replicate is derived from an independent experiment. Total RNA was purified with an RNeasy Plant Mini Kit (Qiagen) according to manufacturer's instructions. RNA-seq libraries were prepared from $1 \mu \mathrm{g}$ total RNA according to TruSeq RNA sample preparation v2 guide (Illumina). Library construction and RNA sequencing was done by the Max-Planck Genome Centre (MPIPZ, Cologne), and produced 21-32 million $100 \mathrm{bp}$ 
reads per sample. RNA-seq reads were mapped to the annotated genome of Arabidopsis thaliana (TAIR10) using TOPHAT2 $(\mathrm{a}=10, \mathrm{~g}=10)(\mathrm{Kim}$ et al, 2013) and transformed into a read count per gene per sample using the htseq-count script ( $\mathrm{s}=$ reverse, $\mathrm{t}=$ exon) in the package HTSeq (Anders et al., 2015). Genes with $<100$ reads across samples were discarded. The count data from the remaining genes were TMM-normalized and $\log _{2}$ transformed using functions 'calcNormFactors' (R package EdgeR; (Robinson et al., 2009)) and 'voom' (R package limma; (Law et al., 2014)). To analyze differential gene expression over time between the different genotypes and treatments, for each analysis we fitted a linear model to the respective $\log _{2}$-transformed count data using the function lmFit (R package limma; (Law et al., 2014)) and subsequently performed moderated t-tests for specific comparisons of interest. In all cases, the resulting $\mathrm{p}$ values were adjusted for false discoveries due to multiple hypothesis testing via the Benjamini-Hochberg procedure. For each comparison, we extracted a set of significantly differentially expressed genes between the tested conditions (adjusted p-value $\leq$ 0.05, $|\log 2 \mathrm{FC}| \geq 1)$. RNA-seq experiments for Pst AvrRps4 and Pst $\Delta$ cor AvrRps4 were performed in separate batches and therefore normalized to Col-0 for the respective treatments to negate potential batch effects. The normalized values were used to generate a heatmap with hierarchial clustering. Circos plot was created using the R package 'Circlize' (Gu et al, 2014), to show the overlap of cluster \#17 with other datasets.

\section{qRT-PCR analysis}

Total RNA was extracted using a Plant RNA kit (Bio-budget). 500 ng total RNA was used for cDNA synthesis (quanta bio) and qRT-PCR analysis was performed using SYBR green master mix. The housekeeping gene $G a p D H$ was used as reference.

\section{Plasmid constructs}

The pENTR/D-TOPO-cEDS1, pENTR/D-TOPO-gEDS1 and pENTR/D-TOPO cPAD4 vectors used for site-directed mutagenesis are previously described (Wagner et al, 2013; Stuttmann et al, 2016). Site-directed mutagenesis on the entry vectors was performed according to the QuikChange II site-directed mutagenesis manual (Agilent). Mutated entry clones were verified by sequencing and recombined into a pAM-PAT-based binary vector backbone by LR reaction.

\section{Generation of Arabidopsis transgenic plants}

Stable transgenic lines were generated by transforming binary expression vectors into Arabidopsis null mutants eds1-2 or pad4-1 sag101-3, as indicated, using Agrobacteriummediated floral dipping.

\section{Yeast two-hybrid assays}

Yeast 2-hybrid (Y2H) assays were performed using the Matchmaker system (Clontech) with strain AH109. Gateway cassettes were cloned into pGADT7 and pGBKT7 plasmids. Newly made PAD4 and EDS1 site-directed EP-domain mutants and the EDS1 ${ }^{\text {LLIF }}$ variant (Wagner et $a l, 2013)$ were recombined into these plasmids by LR reaction. pGAD- and pGBK-containing co-transformants were selected on plates lacking leucine and tryptophan (-LW). Single colonies were re-streaked on plates additionally lacking histidine and adenine (-LWHA) to monitor reporter activation. Yeast growth was recorded after $2-5 \mathrm{~d}$ incubation at $30^{\circ} \mathrm{C}$.

\section{Transient expression in Arabidopsis protoplasts}


Leaf mesophyll protoplasts were prepared from 4-week-old eds1-2 pad4-1 sag101-3 plants and transfections with plasmid DNA were done according to (Yoo et al., 2007). After transfection, protoplasts were incubated at room temperature under weak light $\left(1.5 \mu \mathrm{E} / \mathrm{m}^{2} \mathrm{~s}\right)$ for $16 \mathrm{~h}$. Protoplasts were harvested and IPs performed as described below.

\section{Protein extraction, immunoprecipitation (IP) and immunoblotting}

Total leaf extracts or protoplasts were processed in extraction buffer (50 mM Tris pH 7.5, 150 $\mathrm{mM} \mathrm{NaCl}, 10 \%$ (v/v) glycerol, $2 \mathrm{mM}$ EDTA, $5 \mathrm{mM}$ DTT, protease inhibitor (Roche, 1 tablet per $50 \mathrm{ml}, 0.1 \%$ Triton). Lysates were centrifuged for $15 \mathrm{~min}, 12000 \mathrm{rpm}$ at $4{ }^{\circ} \mathrm{C} .50 \mu \mathrm{l}$ of supernatant was used as input sample. Immunoprecipitations (IPs) were conducted by incubating the input sample with $12 \mu \mathrm{l}$ GFP-TrapA beads (Chromotek) for $2 \mathrm{~h}$ at $4^{\circ} \mathrm{C}$. Beads were collected by centrifugation at $2000 \mathrm{rpm}, 1 \mathrm{~min}$ at $4^{\circ} \mathrm{C}$. Beads were washed three times in extraction buffer and boiled at $95^{\circ} \mathrm{C}$ in $2 \times$ Laemmli buffer for $10 \mathrm{~min}$. Proteins were separated by SDS-PAGE and analyzed by immunoblotting. Antibodies used were $\alpha$-GFP (Sigma Aldrich, 11814460001), $\alpha$-HA (Sigma Aldrich, 11867423001), $\alpha$-FLAG (Sigma Aldrich, F3165). Secondary antibodies coupled to Horseradish Peroxidase (HRP) were used for protein detection on blots (Santa Cruz Biotechnology and Sigma).

\section{SA quantitation}

Free SA was quantified from leaf tissues (70-200 mg fresh weight), of four-week-old plants using a chloroform/methanol extraction and analysed by gas chromatography coupled to a mass spectrometer (GC-MS, Agilent), as described (Straus et al., 2010). Statistical analysis was performed by Student's t-test with multiple testing correction using the Bonferroni method $(\mathrm{p}<0.05)$.

\section{Data Availability}

The RNA-seq data are deposited in the National Center for Biotechnology Information Gene Expression Omnibus (GEO) database with accession number GSE116269.

\section{Acknowledgements}

We thank Kenichi Tsuda and Takaki Maekawa (MPIPZ Cologne) for helpful discussions. This work was supported by The Max-Planck Society and Deutsche Forschungsgemeinschaft (DFG) grants within SFB 635 and SFB 670 (DDB), SFB 680 (DL), and an International MaxPlanck Research School (IMPRS) doctoral fellowship (PvB).

\section{Author contributions}

DDB and JEP designed the study; DDB and PVB performed experiments; BK, DDB and DL analysed the RNA-seq data; DDB and JB generated transgenic plant lines; KN provided structural insight; DDB and JEP wrote the paper with inputs from DL 
bioRxiv preprint doi: https://doi.org/10.1101/362921; this version posted July 5, 2018. The copyright holder for this preprint (which was not certified by peer review) is the author/funder, who has granted bioRxiv a license to display the preprint in perpetuity. It is made available under aCC-BY-NC-ND 4.0 International license.

\section{Conflict of interest}

666 The authors declare no conflict of interest.

667 
668

669

670

671

672

673

674

675

676

677

678

679

680

681

682

683

684

685

686

687

688

689

690

691

692

693

694

695

696

697

698

699

700

701

702

703

704

705

706

707

708

\section{References}

An C, Li L, Zhai Q, You Y, Deng L, Wu F, Chen R, Jiang H, Wang H, Chen Q \& Li C (2017) Mediator subunit MED25 links the jasmonate receptor to transcriptionally active chromatin. Proc. Natl. Acad. Sci. U. S. A. 114:E8930-E8939

Anders S, Pyl PT \& Huber W (2015) HTSeq-A Python framework to work with highthroughput sequencing data. Bioinformatics 31: 166-169

Bartsch M, Bautor J \& Parker JE (2006) Salicylic Acid - Independent ENHANCED DISEASE SUSCEPTIBILITY1 Signaling in Arabidopsis Immunity and Cell Death Is Regulated by the Monooxygenase FMO1 and the Nudix Hydrolase NUDT7. Plant Cell 18: 1038-1051

Berens ML, Berry HM, Mine A, Argueso CT \& Tsuda K (2017) Evolution of Hormone Signaling Networks in Plant Defense. Annu. Rev. Phytopathol. 55: annurev-phyto080516-035544

Birkenbihl RP, Liu S \& Somssich IE (2017) Transcriptional events defining plant immune responses. Curr. Opin. Plant Biol. 38: 1-9

Bonardi V, Tang S, Stallmann A, Roberts M, Cherkis K \& Dangl JL (2011) Expanded functions for a family of plant intracellular immune receptors beyond specific recognition of pathogen effectors. Proc. Natl. Acad. Sci. U. S. A. 108: 16463-8

Brooks DM, Bender CL \& Kunkel BN (2005) The Pseudomonas syringae phytotoxin coronatine promotes virulence by overcoming salicylic acid-dependent defences in Arabidopsis thaliana. Mol. Plant Pathol. 6: 629-639

Cui H, Gobbato E, Kracher B, Qiu J, Bautor J \& Parker JE (2017) A core function of EDS1 with PAD4 is to protect the salicylic acid defense sector in Arabidopsis immunity. New Phytol. 213: 1802-1817

Cui H, Qiu J, Zhou Y, Bhandari D, Bautor J \& Parker JE (2018) Antagonism of transcription factor MYC2 by EDS1/PAD4 complexes bolsters salicylic acid defense in Arabidopsis effector-triggered immunity. Mol. Plant

Cui H, Tsuda K \& Parker JE (2015) Effector-triggered immunity: from pathogen perception to robust defense. Annu. Rev. Plant Biol. 66: 487-511

Dong OX, Tong M, Bonardi V, El Kasmi F, Woloshen V, Wünsch LK, Dangl JL \& Li X (2016) TNL-mediated immunity in Arabidopsis requires complex regulation of the redundant ADR1 gene family. New Phytol. 210: 960-973

Edgar RC (2004) MUSCLE: multiple sequence alignment with high accuracy and high throughput. Nucleic Acids Res. 32: 1792-7

Eulgem T, Weigman VJ, Chang H, Mcdowell JM \& Holub EB (2004) Gene Expression Signatures from Three Genetically Separable Resistance Gene Signaling Pathways for Downy Mildew Resistance. Plant Physiol. 135: 1129-1144

Fenyk S, Townsend PD, Dixon CH, Spies GB, de San Eustaquio Campillo A, Slootweg EJ, Westerhof LB, Gawehns FKK, Knight MR, Sharples GJ, Goverse A, Pålsson L-O, Takken FLW \& Cann MJ (2015) The Potato Nucleotide-binding Leucine-rich Repeat (NLR) Immune Receptor Rx1 Is a Pathogen-dependent DNA-deforming Protein. J. Biol. Chem. 290: $24945-24960$ 
Feys BJ, Moisan LJ, Newman M a \& Parker JE (2001) Direct interaction between the Arabidopsis disease resistance signaling proteins, EDS1 and PAD4. EMBO J. 20: 540011

Feys BJ, Wiermer M, Bhat R a, Moisan LJ, Medina-Escobar N, Neu C, Cabral A \& Parker JE (2005) Arabidopsis SENESCENCE-ASSOCIATED GENE101 stabilizes and signals within an ENHANCED DISEASE SUSCEPTIBILITY1 complex in plant innate immunity. Plant Cell 17: 2601-13

Fu ZQ \& Dong X (2013) Systemic acquired resistance: turning local infection into global defense. Annu. Rev. Plant Biol. 64: 839-63

Gao F, Dai R, Pike SM, Qiu W \& Gassmann W (2014) Functions of EDS1-like and PAD4 genes in grapevine defenses against powdery mildew. Plant Mol. Biol. 86: 381-393

García A V, Blanvillain-Baufumé S, Huibers RP, Wiermer M, Li G, Gobbato E, Rietz S \& Parker JE (2010) Balanced nuclear and cytoplasmic activities of EDS1 are required for a complete plant innate immune response. PLoS Pathog. 6: e1000970

García A V \& Parker JE (2009) Heaven's Gate: nuclear accessibility and activities of plant immune regulators. Trends Plant Sci. 14: 479-87

Geng X, Jin L, Shimada M, Kim MG \& Mackey D (2014) The phytotoxin coronatine is a multifunctional component of the virulence armament of Pseudomonas syringae. Planta 240: $1149-1165$

Gimenez-Ibanez S, Boter M, Fernández-Barbero G, Chini A, Rathjen JP \& Solano R (2014) The bacterial effector HopX1 targets JAZ transcriptional repressors to activate jasmonate signaling and promote infection in Arabidopsis. PLoS Biol. 12: e1001792

Gu Z, Gu L, Eils R, Schlesner M \& Brors B (2014) Circlize implements and enhances circular visualization in R. Bioinformatics 30: 2811-2812

Hatsugai N, Igarashi D, Mase K, Lu Y, Tsuda Y, Chakravarthy S, Wei H, Foley JW, Collmer A, Glazebrook J \& Katagiri F (2017) A plant effector-triggered immunity signaling sector is inhibited by pattern-triggered immunity. EMBO J. 36: 2758-2769

Heidrich K, Wirthmueller L, Tasset C, Pouzet C, Deslandes L \& Parker JE (2011) Arabidopsis EDS1 connects pathogen effector recognition to cell compartment-specific immune responses. Science 334: 1401-4

Hickman R, van Verk MC, Van Dijken AJH, Pereira Mendes M, Vroegop-Vos IA, Caarls L, Steenbergen M, Van Der Nagel I, Wesselink GJ, Jironkin A, Talbot A, Rhodes J, de Vries M, Schuurink RC, Denby K, Pieterse CMJ \& Van Wees SCM (2017) Architecture and Dynamics of the Jasmonic Acid Gene Regulatory Network. Plant Cell: tpc.00958.2016

Inoue H, Hayashi N, Matsushita A, Xinqiong L, Nakayama A, Sugano S, Jiang C-J \& Takatsuji $\mathrm{H}$ (2013) Blast resistance of CC-NB-LRR protein $\mathrm{Pb} 1$ is mediated by WRKY45 through protein-protein interaction. Proc. Natl. Acad. Sci. U. S. A. 110: 9577-82

Jacob F, Kracher B, Mine A, Seyfferth C, Blanvillain-Baufumé S, Parker JE, Tsuda K, SchulzeLefert P \& Maekawa T (2018) A dominant-interfering camta3 mutation compromises primary transcriptional outputs mediated by both cell surface and intracellular immune receptors in Arabidopsis thaliana. New Phytol. 217: 1667-1680

Jacob F, Vernaldi S \& Maekawa T (2013) Evolution and Conservation of Plant NLR Functions. 
Jiang S, Yao J, Ma K-W, Zhou H, Song J, He SY \& Ma W (2013) Bacterial Effector Activates Jasmonate Signaling by Directly Targeting JAZ Transcriptional Repressors. PLoS Pathog. 9: e1003715

Jirage D, Tootle TL, Reuber TL, Frost LN, Feys BJ, Parker JE, Ausubel FM \& Glazebrook J (1999) Arabidopsis thaliana PAD4 encodes a lipase-like gene that is important for salicylic acid signaling. Proc. Natl. Acad. Sci. U. S. A. 96: $13583-8$

Jones JDG, Vance RE \& Dangl JL (2016) Intracellular innate immune surveillance devices in plants and animals. Science (80-. ). 354: aaf6395-aaf6395

Kazan K \& Lyons R (2014) Intervention of Phytohormone Pathways by Pathogen Effectors. Plant Cell 26: 2285-2309

Kazan K \& Manners JM (2013) MYC2: The master in action. Mol. Plant 6: 686-703

Kim D, Pertea G, Trapnell C, Pimentel H, Kelley R \& Salzberg SL (2013) Comment on “ TopHat2 : accurate alignment of transcriptomes in the presence of insertions, deletions and gene fusions ': 0-9

Kim Y, Tsuda K, Igarashi D, Hillmer RA, Sakakibara H, Myers CL \& Katagiri F (2014) Mechanisms underlying robustness and tunability in a plant immune signaling network. Cell Host Microbe 15: 84-94

Kunkel BN, Bent AF, Dahlbeck D, Innes RW \& Staskawicz BJ (1993) RPS2, an Arabidopsis disease resistance locus specifying recognition of Pseudomonas syringae strains expressing the avirulence gene avrRpt2. Plant Cell 5: 865-75

Law CW, Chen Y, Shi W \& Smyth GK (2014) Voom: Precision weights unlock linear model analysis tools for RNA-seq read counts. Genome Biol. 15: 1-17

Le Roux C, Huet G, Jauneau A, Camborde L, Trémousaygue D, Kraut A, Zhou B, Levaillant M, Adachi H, Yoshioka H, Raffaele S, Berthomé R, Couté Y, Parker JE \& Deslandes L (2015) A Receptor Pair with an Integrated Decoy Converts Pathogen Disabling of Transcription Factors to Immunity. Cell 161: 1074-1088

Li X, Clarke JD, Zhang Y \& Dong X (2001) Activation of an EDS1-mediated R-gene pathway in the snc1 mutant leads to constitutive, NPR1-independent pathogen resistance. Mol. Plant. Microbe. Interact. 14: 1131-1139

Mine A, Seyfferth C, Kracher B, Berens M, Becker D \& Tsuda K (2018) The Defense Phytohormone Signaling Network Enables Rapid, High-amplitude Transcriptional Reprogramming During Effector-Triggered Immunity. Plant Cell

Muskett PR, Kahn K, Austin MJ, Moisan LJ, Sadanandom A, Shirasu K, Jones JDG \& Parker JE (2002) Exerts Rate-Limiting Control of. Plant Cell 14: 979-992

Navarro L, Zipfel C, Rowland O, Keller I, Robatzek S, Boller T \& Jones JDG (2004) The Transcriptional Innate Immune Response to flg22 . Interplay and Overlap with Avr GeneDependent Defense Responses and Bacterial Pathogenesis 1 [ w ]. 135: 1113-1128

Nobori T, Velásquez AC, Wu J, Kvitko BH, Kremer JM, Wang Y, He SY \& Tsuda K (2018) Transcriptome landscape of a bacterial pathogen under plant immunity. Proc. Natl. Acad. Sci. 20: 180052915 
Parker JE, Holub EB, Frost LN, Falk A, Gunn ND \& Danielsa MJ (1996) Characterization of edsl, a Mutation in Arabidopsis Suppressing Resistance to Peronospora parasííca Specified by Severa1 Different RPP Genes. 8: 2033-2046

Pieterse CMJ, Van der Does D, Zamioudis C, Leon-Reyes A \& Van Wees SCM (2012) Hormonal Modulation of Plant Immunity. Annu. Rev. Cell Dev. Biol. 28: 489-521

Rentel MC, Leonelli L, Dahlbeck D, Zhao B \& Staskawicz BJ (2008) Recognition of the Hyaloperonospora parasitica effector ATR13 triggers resistance against oomycete, bacterial, and viral pathogens. Proc Natl Acad Sci U S A 105: 1091-1096

Rietz S, Stamm A, Malonek S, Wagner S, Becker D, Medina-Escobar N, Vlot a C, Feys BJ, Niefind K \& Parker JE (2011) Different roles of Enhanced Disease Susceptibility1 (EDS1) bound to and dissociated from Phytoalexin Deficient4 (PAD4) in Arabidopsis immunity. New Phytol. 191: 107-19

Robinson MD, McCarthy DJ \& Smyth GK (2009) edgeR: A Bioconductor package for differential expression analysis of digital gene expression data. Bioinformatics 26: 139140

Sarris PF, Duxbury Z, Huh SU, Ma Y, Segonzac C, Sklenar J, Derbyshire P, Cevik V, Rallapalli G, Saucet SB, Wirthmueller L, Menke FLH, Sohn KH \& Jones JDG (2015) A Plant Immune Receptor Detects Pathogen Effectors that Target WRKY Transcription Factors. Cell 161: 1089-1100

Saucet SB, Ma Y, Sarris PF, Furzer OJ, Sohn KH \& Jones JDG (2015) Two linked pairs of Arabidopsis TNL resistance genes independently confer recognition of bacterial effector AvrRps4. Nat. Commun. 6: 1-12

Schön M, Töller A, Diezel C, Roth C, Westphal L, Wiermer M \& Somssich IE (2013) Analyses of wrky18 wrky40 Plants Reveal Critical Roles of SA/EDS1 Signaling and IndoleGlucosinolate Biosynthesis for Golovinomyces orontii Resistance and a Loss-of Resistance Towards Pseudomonas syringae pv. tomato AvrRPS4. Mol. Plant. Microbe. Interact. 26: 758-67

Seyfferth C \& Tsuda K (2014) Salicylic acid signal transduction: the initiation of biosynthesis, perception and transcriptional reprogramming. Front. Plant Sci. 5: 1-10

Sinapidou E, Williams K, Nott L, Bahkt S, Tör M, Crute I, Bittner-Eddy P \& Beynon J (2004) Two TIR:NB:LRR genes are required to specify resistance to Peronospora parasitica isolate Cala2 in Arabidopsis. Plant J. 38: 898-909

Stuttmann J, Hubberten H-M, Rietz S, Kaur J, Muskett P, Guerois R, Bednarek P, Hoefgen R \& Parker JE (2011) Perturbation of Arabidopsis Amino Acid Metabolism Causes Incompatibility with the Adapted Biotrophic Pathogen Hyaloperonospora arabidopsidis. Plant Cell 23: 2788-2803

Stuttmann J, Peine N, Garcia A V., Wagner C, Choudhury SR, Wang Y, James GV, Griebel T, Alcázar R, Tsuda K, Schneeberger K \& Parker JE (2016) Arabidopsis thaliana DM2h (R8) within the Landsberg RPP1-like Resistance Locus Underlies Three Different Cases of EDS1-Conditioned Autoimmunity. PLoS Genet. 12: 1-29

Sun T, Li Y, Zhang Q, Ding Y, Zhang Y \& Zhang Y (2015) ChIP-seq reveals broad roles of SARD1 and CBP60g in regulating plant immunity. Nat. Commun. 6: 1-12

Tao Y, Xie Z, Chen W, Glazebrook J, Chang H, Han B, Zhu T, Zou G \& Katagiri F (2003) 
Quantitative Nature of Arabidopsis Responses during Compatible and Incompatible Interactions with the Bacterial Pathogen Pseudomonas syringae. Plant Cell 15: 317-330

Tsuda K, Mine A, Bethke G, Igarashi D, Botanga CJ, Tsuda Y, Glazebrook J, Sato M \& Katagiri F (2013) Dual regulation of gene expression mediated by extended MAPK activation and salicylic acid contributes to robust innate immunity in Arabidopsis thaliana. PLoS Genet. 9: e1004015

Tsuda K, Sato M, Stoddard T, Glazebrook J \& Katagiri F (2009) Network properties of robust immunity in plants. PLoS Genet. 5: e1000772

Tsuda K \& Somssich IE (2015) Transcriptional networks in plant immunity. New Phyto. 206: $930-947$

Venugopal SC, Jeong R-D, Mandal MK, Zhu S, Chandra-Shekara a C, Xia Y, Hersh M, Stromberg AJ, Navarre D, Kachroo A \& Kachroo P (2009) Enhanced disease susceptibility 1 and salicylic acid act redundantly to regulate resistance gene-mediated signaling. PLoS Genet. 5: e1000545

Wagner S, Stuttmann J, Rietz S, Guerois R, Brunstein E, Bautor J, Niefind K \& Parker JE (2013) Structural Basis for Signaling by Exclusive EDS1 Heteromeric Complexes with SAG101 or PAD4 in Plant Innate Immunity. Cell Host Microbe 14: 619-30

Wang W, Barnaby JY, Tada Y, Li H, Tör M, Caldelari D, Lee DU, Fu XD \& Dong X (2011) Timing of plant immune responses by a central circadian regulator. Nature 470: $110-115$

Wiermer M, Feys BJ \& Parker JE (2005) Plant immunity: the EDS1 regulatory node. Curr. Opin. Plant Biol. 8: 383-9

Wildermuth MC, Dewdney J, Wu G \& Ausubel FM (2001) Isochorismate synthase is required to synthesize salicylic acid for plant defence. Nature 414: 562-565

Williams SJ, Sohn KH, Wan L, Bernoux M, Sarris PF, Segonzac C, Ve T, Ma Y, Saucet SB, Ericsson DJ, Casey LW, Lonhienne T, Winzor DJ, Zhang X, Coerdt A, Parker JE, Dodds PN, Kobe B \& Jones JDG (2014) Structural basis for assembly and function of a heterodimeric plant immune receptor. Science 344: 299-303

Wirthmueller L, Zhang Y, Jones JDG \& Parker JE (2007) Nuclear Accumulation of the Arabidopsis Immune Receptor RPS4 Is Necessary for Triggering EDS1-Dependent Defense. Curr. Biol. 17: 2023-2029

Yang L, Teixeira PJPL, Biswas S, Finkel OM, He Y, Salas-Gonzalez I, English ME, Epple P, Mieczkowski P \& Dangl JL (2017) Pseudomonas syringae Type III Effector HopBB1 Promotes Host Transcriptional Repressor Degradation to Regulate Phytohormone Responses and Virulence. Cell Host Microbe 21: 156-168

Yoo S-D, Cho Y-H \& Sheen J (2007) Arabidopsis mesophyll protoplasts: a versatile cell system for transient gene expression analysis. Nat. Protoc. 2: 1565-72

Yue J-X, Meyers BC, Chen J-Q, Tian D \& Yang S (2012) Tracing the origin and evolutionary history of plant nucleotide-binding site-leucine-rich repeat (NBS-LRR) genes. New Phytol. 193: 1049-63

Zhang F, Yao J, Ke J, Zhang L, Lam VQ, Xin X-F, Zhou XE, Chen J, Brunzelle J, Griffin PR, Zhou M, Xu HE, Melcher K \& He SY (2015a) Structural basis of JAZ repression of MYC transcription factors in jasmonate signalling. Nature 
Zhang L, Yao J, Withers J, Xin X-F, Banerjee R, Fariduddin Q, Nakamura Y, Nomura K, Howe GA, Boland W, Yan H \& He SY (2015b) Host target modification as a strategy to counter pathogen hijacking of the jasmonate hormone receptor. Proc. Natl. Acad. Sci. U. S. A.: 201510745

Zhang X, Dodds PN \& Bernoux M (2017) What Do We Know About NOD-Like Receptors in Plant Immunity? Annu. Rev. Phytopathol. 55: 205-229

Zheng X-Y, Spivey NW, Zeng W, Liu P-P, Fu ZQ, Klessig DF, He SY \& Dong X (2012) Coronatine promotes Pseudomonas syringae virulence in plants by activating a signaling cascade that inhibits salicylic acid accumulation. Cell Host Microbe 11: 587-96

Zhou M, Lu Y, Bethke G, Harrison BT, Hatsugai N, Katagiri F \& Glazebrook J (2018) WRKY70 prevents axenic activation of plant immunity by direct repression of SARD1. New Phytol. 217: 700-712

Zhou N, Tootle TL, Tsui F, Klessig DF \& Glazebrook J (1998) PAD4 functions upstream from salicylic acid to control defense responses in Arabidopsis. Plant Cell 10: 1021-30 


\section{Figure 1. Residues lining the EDS1 heterodimer cavity mediate immunity signalling}

895

896

897

898

899

900

901

902

903

904

905

906

907

908

909

910

911

912

913

914

915

916

917

918

919

920

921

922

923

924

925

926

927

928

929

930

931

932

933

934

935

A.Crystal structure of EDS1 (blue) - SAG101 (green) showing heterodimer formation chiefly driven by the partner lipase-like domains (light tones) and producing a cavity (magenta mesh) formed by the EP-domains. In the zoomout, two conserved EDS1 arginine residues lining the cavity are depicted as sticks (brown). B. RPP2 resistance phenotypes of two-week-old control and transgenic lines expressing YFP-cEDS1 and R493A. Hpa EMWA1 infected leaves were stained with trypan blue at $5 \mathrm{dpi}$. Scale bar represents $100 \mu \mathrm{m}$. Images are representative of 24 leaves from two different experiments. HR, hypersensitive response; fh, pathogen free hyphae. C. Four-week old Arabidopsis plants of the indicated genotypes were infiltrated with Pst AvrRps4 (OD600 - 0.0005) and bacterial titers determined at 0 and 3 dpi. Bars represent mean of four biological replicates \pm SE. Differences between genotypes were determined using ANOVA (Tukey's HSD, p <0.005). Similar results were obtained in three independent experiments. D. Homology model of EDS1 (blue) - PAD4 (green). Conserved residues in the EP-domains of EDS1 (magenta) and PAD4 (purple) are represented as spheres. EDS1 residues line the heterodimer cavity while PAD4 residues are not part of the cavity. The zoomout shows ionic and hydrogen bonds formed by $\mathrm{EDS}^{\mathrm{R} 493}$ and the equivalent arginine residue in PAD4 ${ }^{\mathrm{R} 420}$ with neighbouring residues. E. RPP4 resistance phenotypes of two-week-old control and homozygous transgenic lines expressing wild-type EDS1, PAD4 and mutated arginine variants. Hpa EMWA1 infected leaves were stained with trypan blue at 5 dpi. Scale bar represents 100 $\mu \mathrm{m}$. The PAD4 R420A image is representative of 18 independent transgenic $\left(\mathrm{T}_{1}\right)$ plants. HR, hypersensitive response; fh, pathogen free hyphae.

\section{Figure 2. EDS1 ${ }^{\text {R493A }}$ delays TNL transcriptional reprogramming}

A. Four-week-old plants were infiltrated with Pst AvrRps4 (OD600-0.005) and free SA was quantified at 0,8 and 24 hpi. Bars represent means \pm SE of four biological replicates. Differences between genotypes were analysed using t-test (Bonferroni corrected, $\mathrm{p}<0.05$ ). Similar results were obtained in two independent experiments. B. Four-week-old plants were infiltrated with $10 \mathrm{mM} \mathrm{MgCl} 2$ (mock) or Pst AvrRps4 (OD $600-0.005$ ), and leaf samples collected at 24 hpi. PRl transcripts were measured using qRT-PCR and normalized to GapDH. Bars represent means \pm SE of 3 biological replicates. Difference between genotypes were analysed using t-test (Bonferroni corrected, $\mathrm{p}<0.05$ ). C. A 2-dimensional scatter plot comparing R493A vs eds1-2 and R493A vs cEDS1 at 8 and 24 hpi with Pst AvrRps4. Plots depict differentially expressed genes (DEG) between R493A vs eds1-2 (pink dots) and R493A vs cEDS1 (blue dots). DEG represented were filtered with a $\left|\log _{2} \mathrm{FC}\right| \geq 1, \mathrm{FDR} \leq 0.05$.

\section{Figure 3. EDS1 ${ }^{\mathrm{R} 493 A}$ fails to antagonize bacterial COR-stimulated MYC2 in TNL ETI}

A. Four-week-old Arabidopsis plants of the indicated genotypes were infiltrated with Pst AvrRps4 or Pst $\Delta$ Cor AvrRps4 (OD $600-0.0005)$. Bacterial titers were determined at 0 and 3 dpi. No significant difference was observed between lines and treatments at 0 dpi. Bars represent mean of four biological replicates \pm SE. Differences between genotypes were analysed using ANOVA (Tukey's HSD, p-value <0.005). Similar results were obtained in three independent experiments. B. Four-week-old Arabidopsis plants of the indicated genotypes were infiltrated with Pst AvrRps4 (OD 600 - 0.0005). Bacterial titers were determined at 0 and 3 dpi. No significant differences were observed at 0 dpi. Bars represent mean of four biological 
replicates \pm SE. Differences between genotypes were analysed using ANOVA (Tukey's HSD, p-value <0.005). Similar results were obtained in three independent experiments. C. The indicated genotypes were infected with Hpa EMWA1 and oomycete sporulation was quantified at 5 dpi. Data from three independent experiments were combined and differences between genotypes analysed using ANOVA (Tukey's HSD, $\mathrm{p}<0.005$ ).

\section{Figure 4. Delayed immune signalling in R493A mutant plants is independent of COR}

A. Four-week-old plants were infiltrated with $10 \mathrm{mM} \mathrm{MgCl} 2$ (mock), Pst AvrRps 4 or Pst $\Delta$ Cor AvrRps 4 and free SA was quantified at 8 hpi. Bars represent means \pm SE of three biological replicates. Difference between genotypes were analysed using t-test (Bonferroni corrected, $\mathrm{p}<0.05)$. Similar results were observed in three independent experiments. B. A multidimension scaling (MDS) plot of differentially expressed genes showing R493A transcriptional changes at 8 (open symbols) and 24 hpi (closed symbols). Encircled samples of eds 1-2 (pink) and R493A (purple) highlight transcriptional trends with and without bacterial COR in Pst AvrRps4-triggered immunity. C. A heatmap depicting DEG at 8 and 24 hpi normalized to Col $(\mathrm{p}<0.05)$ after hierarchical clustering. Samples were harvested at 8 and 24 hpi with Pst AvrRps 4 (Avr) and Pst $\triangle$ Cor AvrRps 4 ( $\triangle$ Cor). Cluster \#17 contains genes that are upregulated at 8 hpi with Pst $\triangle$ Cor AvrRps4 but not Pst AvrRps4 in R493A only. Expansion (right) highlights a subset of $N L R$ and $W R K Y$ genes in cluster \#17 that are involved in immunity whose expression is antagonized by COR in R493A (see Table S3). D. Circos plot showing overlap of 383 genes in cluster \#17 with genes regulated by JA and BTH from other studies (Hickmann et al.,2017; Yang et al.,2017). Genes differentially expressed in cluster \#17 and other datasets are marked by connecting lines. Genes repressed by JA and induced by BTH (red and green lines converging to yellow, 113); genes repressed by JA and not expressed by BTH (green lines, 19); genes induced by BTH and not expressed in JA dataset (red lines, 169).

\section{Figure 5. A positive charge at EDS1 ${ }^{\mathrm{R} 493}$ is essential for TNL immunity}

A. $R P P 2$ resistance phenotypes of two-week-old control and Arabidopsis transgenic lines expressing cEDS 1 and R493 mutants, as indicated. Hpa EMWA1 infected leaves were stained with trypan blue at 5 dpi. Scale bar represents $100 \mu \mathrm{m}$. Each image is representative of $>18$ leaves from two independent experiments. HR, hypersensitive response; fh, pathogen free hyphae. B. Four-week-old Arabidopsis plants of the indicated genotypes were infiltrated with Pst AvrRps 4 and Pst $\Delta$ Cor AvrRps 4 (OD 600 - 0.0005). Bacterial titers were determined at 3 dpi. Bars represent means of three biological replicates \pm SE. Differences between genotypes were analysed using ANOVA (Tukey's HSD, $\mathrm{p}<0.05$ ). Similar results were obtained in three independent experiments. C. Four-week-old plants were infiltrated with Pst AvrRps4 or Pst $\Delta$ Cor AvrRps 4 and free SA was quantified at 8 and 24 hpi. Bars represent means \pm SE of three biological replicates. Differences between genotypes within treatment were analysed using Student's t-test (Bonferroni corrected, $* * *-p<0.05$ ) relative to Col. Similar results were obtained in two independent experiments.

\section{Figure 6. EDS1 ${ }^{\mathrm{R} 493}$ signals in TNL and CNL (RPS2) immunity}

A. Four-week-old Arabidopsis plants of the indicated genotypes were infiltrated with Pst DC3000 and Pst $\Delta$ Cor (OD600 - 0.0005). Bacterial titers were determined at 0 and 3 dpi. No significant difference was observed between lines and treatments at 0 dpi. Bars represent mean of four biological replicates \pm SE. Differences between genotypes were analysed using 
1002

1003

1004

1005

1006

1007

1008

1009

1010

1011

1012

1013

1014

1015

1016

1017

1018

1019

ANOVA (Tukey's HSD, $\mathrm{p}<0.005$ ). Similar results were obtained in three independent experiments. B. Four-week-old Arabidopsis plants of the indicated genotypes were infiltrated with Pst $\Delta$ Cor AvrRps4 (OD600 - 0.0005). Bacterial titers were determined at 0 and 3 dpi. No significant difference was observed at 0 dpi. Bars represent mean of four biological replicates \pm SE. Differences between genotypes were analysed using ANOVA (Tukey's HSD, $p<0.005$ ). Similar results were obtained in three independent experiments. C. Four-week-old Arabidopsis plants of the indicated genotypes were infiltrated with Pst AvrRpt2 (OD600 - 0.0005). Bacterial titers were determined at 0 and $3 \mathrm{dpi}$. No significant difference was observed at $0 \mathrm{dpi}$. Bars represent mean of four biological replicates \pm SE. Differences between genotypes were analysed using ANOVA (Tukey's HSD, $\mathrm{p}<0.005$ ). Similar results were obtained in three independent experiments.

\section{Figure 7. A model of EDS1 signalling branches in RRS1S RPS4 ETI}

A three-pronged ETI signalling model derives from comparisons of wild-type EDS1, EDS $1^{\mathrm{R} 493 \mathrm{~A}}$ and $e d s 1-2$ phenotypes in this study. Three interconnected EDS1 functions contribute to robust TNL ETI. 1) TNL-activated wild-type EDS1 effectively counters COR antagonism of immunity gene expression via MYC2. The defective EDS1 EP-domain mutant R493A is susceptible in TNL ETI against Pst AvrRps4 due to its inability to counter COR/MYC2 antagonism. 2) EDS1 boosts SA accumulation independently of antagonising MYC2 while EDS1 ${ }^{\mathrm{R} 493 \mathrm{~A}}$ delays SA accumulation (dashed lines) independently of COR repressive effects. 3) An additional EDS1 branch (X) in TNL (RRS1S RPS4) ETI is revealed after removing ICSI/SA and COR effects. The EDS1 EP-domain, and more specifically $\mathrm{EDS} 1^{\mathrm{R} 493}$, is also necessary for this resistance branch. The nature of branch $\mathrm{X}$ requires further study.

\section{Supplementary figure legends}

\section{Figure S1}

A. Crystal structure of EDS1 (blue) - SAG101 (green) heterodimer with cavity formed by the EP-domains (magenta mesh) highlighted. Conserved positively charged residues lining the cavity are depicted as brown sticks. B. Y2H interactions between activation domain (AD) fusions of EDS1 variants and PAD4 (BD) binding domain fusions. The EDS1-LLIF mutant which does not bind PAD4 was used as a negative control. Yeast viability (-LW) and protein interaction (-LWAH) are shown in the GAL4 matchmaker Y2H system. C. Summary of TNL (RPP4) complementation assay in $\mathrm{T}_{1}$ plants expressing EDS1-YFP EP-domain mutants. For each EDS1 mutant line, individual BASTA-resistant $\mathrm{T}_{1}$ seedlings were monitored for TNLtriggered resistance to Hpa EMWA1 (at 5 dpi). Seedlings showing conidospores on leaves were scored as disease susceptible. Numbers of resistant / total plants tested is shown. Nontransgenic controls for RPP4 resistance Col-0 and eds $1-2$ were not treated with BASTA. D. Conservation of EDS1 EP-domain residues across EDS1 orthologs. Sequences were aligned using MUSCLE (Edgar, 2004) and positions corresponding to K478 and R493 are highlighted, including the characteristic "EPLDIA" conserved motif of the EP-domain.

\section{Figure S2}


A. cEDS1-FLAG variants transiently co-expressed with cPAD4-YFP in eds1-2 pad4-1 sag101-3 protoplasts (input) were immunoprecipitated (IP) using $\alpha$-GFP beads. Coimmunoprecipitated FLAG-tagged cEDS1 variants were detected using $\alpha$-Flag antibodies. EDS1-LLIF which (migrates higher) does not bind PAD4 was used as a negative control. B. Confocal images of four-week-old leaves of transgenic eds1-2 lines expressing YFP-tagged EDS1 or R493A, showing nucleocytoplasmic localization. Images were taken at 24 hpi with Pst AvrRps4. Images are representative of $>30$ cells per line. Scale bar $=25 \mu \mathrm{m}$. C. Four-weekold Arabidopsis plants of the indicated genotypes were infiltrated with Pst AvrRps4 (OD 600 $0.0005)$ and bacterial titers determined at 0 and 3dpi. No significant difference was observed at 0 dpi. Bars represent mean of three biological replicates \pm SE. Differences between genotypes were analysed using ANOVA (Tukey's HSD, $\mathrm{p}<0.005$ ). Similar results were obtained in three independent experiments. D. Accumulation of EDS1-YFP protein in mock and Pst AvrRps4 (24 hpi) treated plants of cEDS1, gEDS1 and respective R493A mutants on immunoblots probed using $\alpha$-GFP antibody.

\section{Figure S3}

A. Comparison of expression profiles of genes regulated by ICS1/SA/(Tsuda et al., 2013) and repressed by JA (Hickmann et al., 2017; selected clusters (\#15, 18, 19, 20, 23, 24) were chosen based on differential expression profile between cEDS1 and eds1-2) with our study using Pst AvrRps 4 and Pst $\Delta$ Cor AvrRps 4 ( $\Delta$ Cor). Bar plots are coloured based on genoytpe with a light tone for the $\Delta$ Cor data. Statistical differences between genotypes were analysed within treatment using the Kruskal-Nemenyi test $(\mathrm{p}<0.001)$ as indicated by different annotations. B. A Venn diagram presenting the overlap between genes in cluster \#17 for AvrRps4 ETI at 8hpi, genes that are specifically regulated in AvrRpt2-triggered ETI (ETI-specific genes) (Mine et al., 2018), DEG at 4 hpi with Pst AvrRpt2 (Pst AvrRpt2 - 4 hpi) (Mine et al., 2018) and DEG at $5 \mathrm{~h}$ with estradiol-induced AvrRpt2 (ED-AvrRpt2-5hpi) (Hatsugai et al., 2017).

\section{Figure S4}

A. Confocal images of transiently expressed FLAG-tagged cEDS1 and R493 mutant variants in $N$. benthamiana showing nucleocytoplasmic localization. White arrowheads depict nuclei and chloroplasts fluoresce red. Images are representative of $>20$ cells/variant at $3 \mathrm{~d}$ after Agroinfiltration. Scale bar $=20 \mu \mathrm{m}$. B. EDS1-FLAG variants transiently expressed with PAD4YFP in eds1-2 pad4-1 protoplasts were immunoprecipitated using $\alpha$-GFP beads. Coimmunoprecipitated EDS1 variants were detected using $\alpha$-FLAG antibodies. EDS $1{ }^{\text {LLIF }}$-FLAG and YFP-FLAG were used as negative controls in the IP. C. EDS1-FLAG variants, as used in B. were transiently expressed with PAD4-YFP and strep-HA-MYC2 (SH-MYC2) in eds1-2 pad4-1 sag 101-3 protoplasts. PAD4-YFP was immunoprecipitated using $\alpha$-GFP beads. Coimmunoprecipitated FLAG-tagged EDS1 variants and SH-MYC2 were detected on immunoblots using $\alpha$-FLAG and $\alpha$-HA antibodies, respectively. EDS1 ${ }^{\text {LLIF }}$-FLAG and YFPFLAG were used as negative controls. IPs were repeated three times with similar results.

\section{Figure S5}

A. Expression of EDS1, PAD4 and ICS1 in EDS1 transgenic lines, as indicated, at 8 hpi with Pst AvrRps4 or Pst $\triangle C O R$ AvrRps4, measured by qRT-PCR. Values were normalized to the house-keeping gene $G a p D H$. Bars represent means \pm SE calculated from three independent experiments. Differences between genotypes were calculated using ANOVA (Tukey's HSD, 


\section{Supplementary data tables}

1070 Table S1. List of DEG between R493A and eds1-2 at 8 hpi with Pst AvrRps4.

1071 Table S2. Summary of total DEG between Pst AvrRps4 and Pst $\Delta$ Cor AvrRps4 treatments for 1072 each genotype at 8 hpi and 24 hpi.

1073 Table S3. List of genes in cluster \#17 (associated with Fig. 4C, D).

1074 Table S4. Gene Ontology (GO) enrichment of cluster \#17 genes. GO enrichment was obtained 1075 using the panther database.

1076 Table S5. Overlapping genes between cluster \#17, BTH-regulated genes and JA-regulated 1077 genes (associated with Fig. 4D).

1078 Table S6. List of primers used in this study. 
A
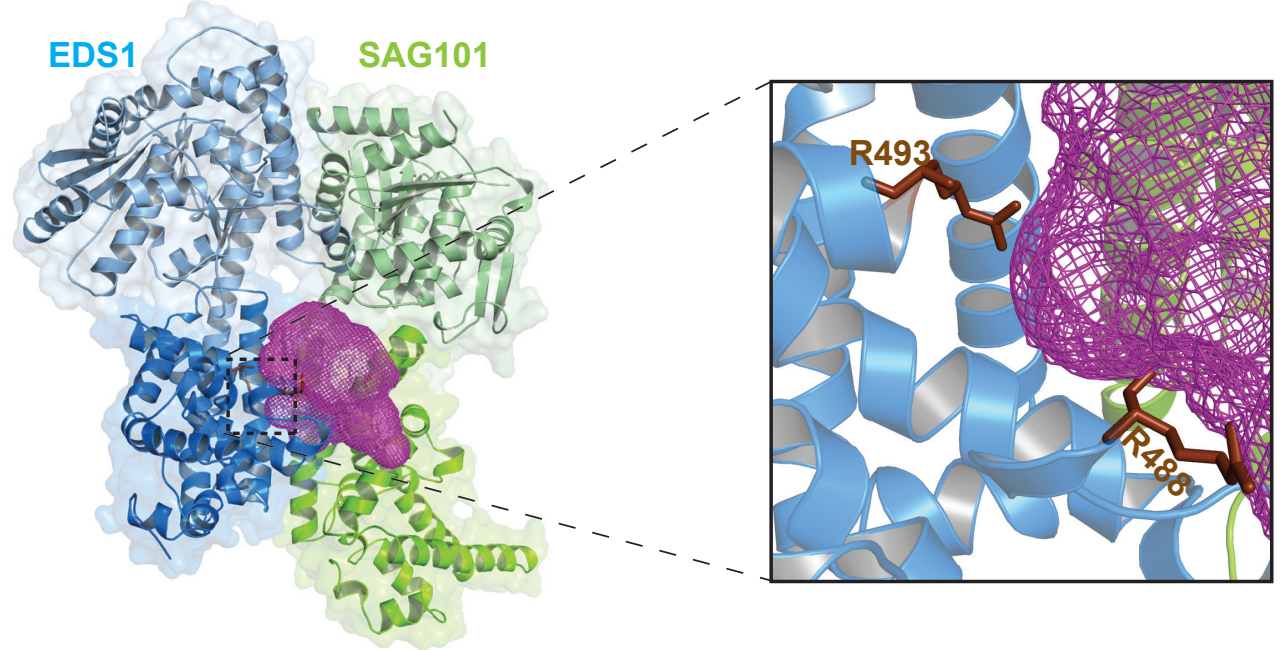

B

Hpa CALA2

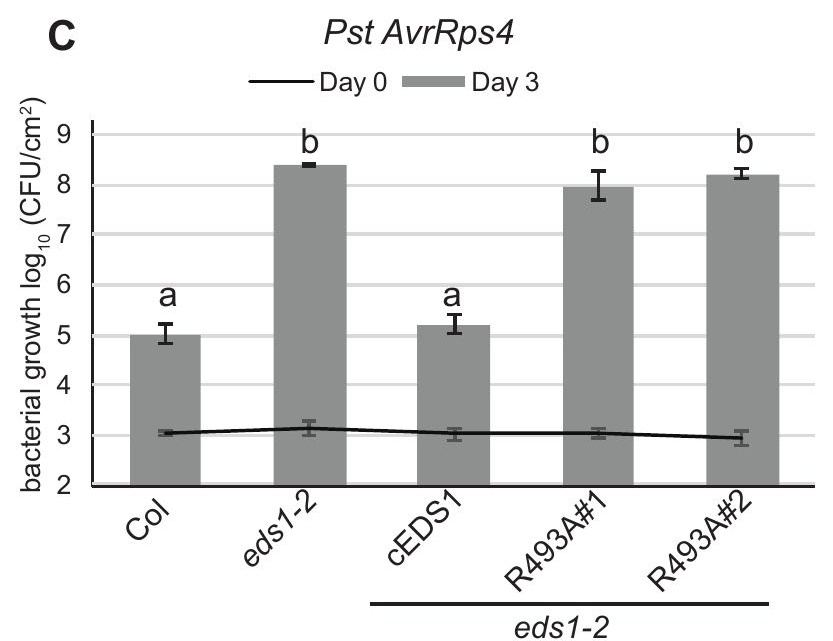

D
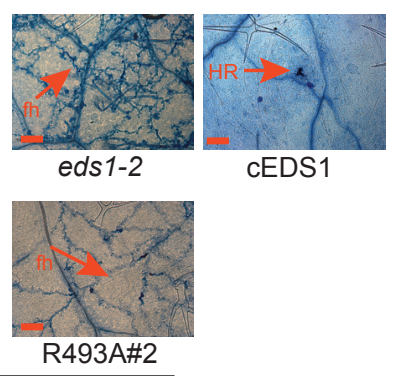

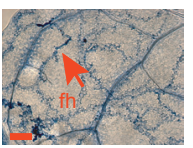

R493A\#1

eds1-2

$\mathbf{E}$

Hpa EMWA1

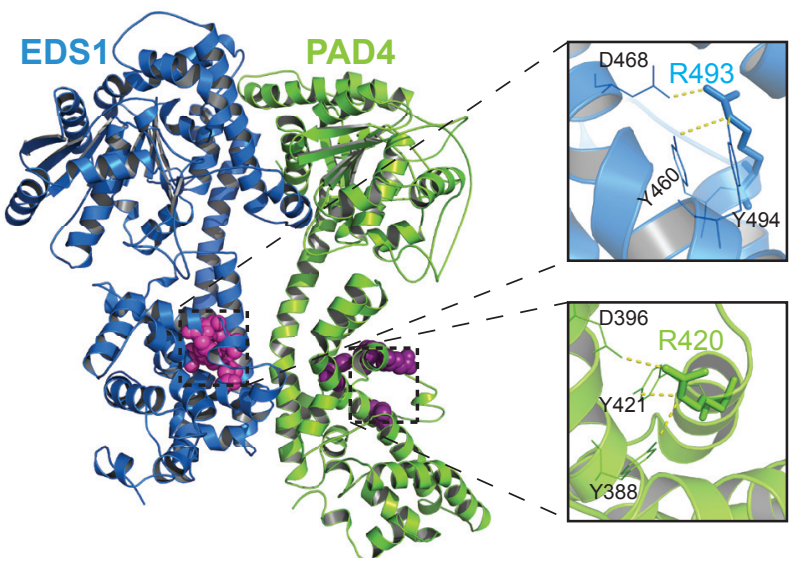

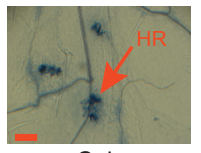

Col
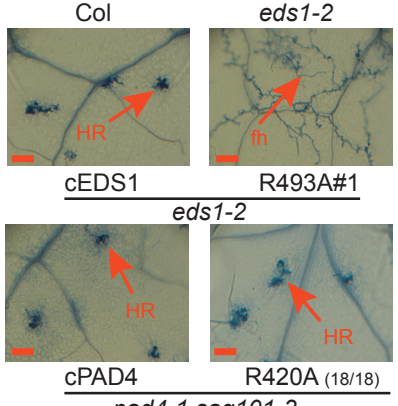

Figure 1

Residues lining the EDS1 heterodimer cavity mediate immunity signalling

A. Crystal structure of EDS1 (blue) - SAG101 (green), showing heterodimer formation chiefly driven by the lipase-like domains (light tones) producing a cavity (magenta mesh) formed by the EP-domains. In the zoomout, two conserved EDS1 arginine residues lining the cavity are depicted as sticks (brown). B. RPP2 resistance phenotypes of two-week old control and transgenic lines expressing YFP-cEDS1 and R493A. Hpa EMWA1 infected leaves were stained with trypan blue at $5 \mathrm{dpi}$. Scale bar represents $100 \mu \mathrm{m}$. Images are representative of 24 leaves from two different experiments. HR-hypersensitive response; fh-pathogen free hyphae. C. Four-week old Arabidopsis plants of the indicated genotypes were infiltrated with Pst AvrRps4 (OD600 - 0.0005) and bacterial titers determined at 0 and 3 dpi. Bars represent mean of four biological replicates \pm standard error. Differences between genotypes were analysed using ANOVA (Tukey's HSD, $\mathrm{p}<0.005$ ). Similar results were obtained in three independent experiments. D. Homology model of EDS1 (blue) - PAD4 (green). Conserved residues in the EP-domains of EDS1(magenta) and PAD4 (purple) are represented as spheres. EDS1 residues line the heterodimer cavity while PAD4 residues face away from the interfaceand not part of the cavity. The Zoomout shows ionic and hydrogen bonds formed by EDS1-R493 and the equivalent arginine residue in PAD4-R420 form similar interactions with neighbouring residues. E. RPP4 resistance phenotypes of two-week-old control and homozygous transgenic lines expressing wild-type EDS1, PAD4 and mutated arginine variants. Hpa EMWA1 infected leaves were stained with trypan blue at 5 dpi. Scale bar represents $100 \mu \mathrm{m}$. The PAD4 R420A image is representative of 18 independent transgenic (T1) plants. HR-hypersensitive response; fh-pathogen free hyphae. 
A

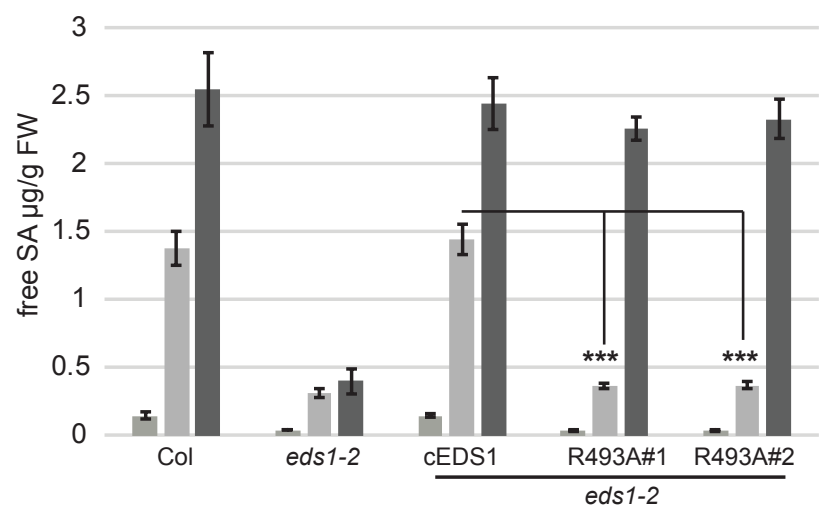

B

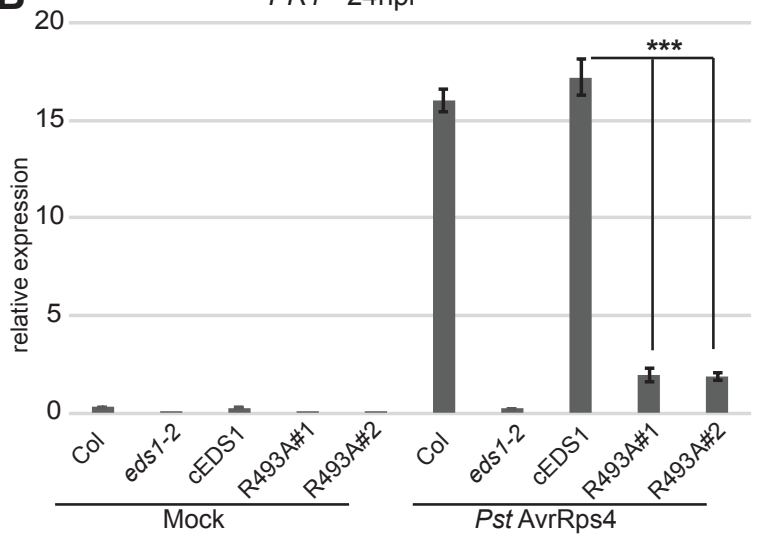

C

8 h DEG

$24 \mathrm{~h}$ DEG
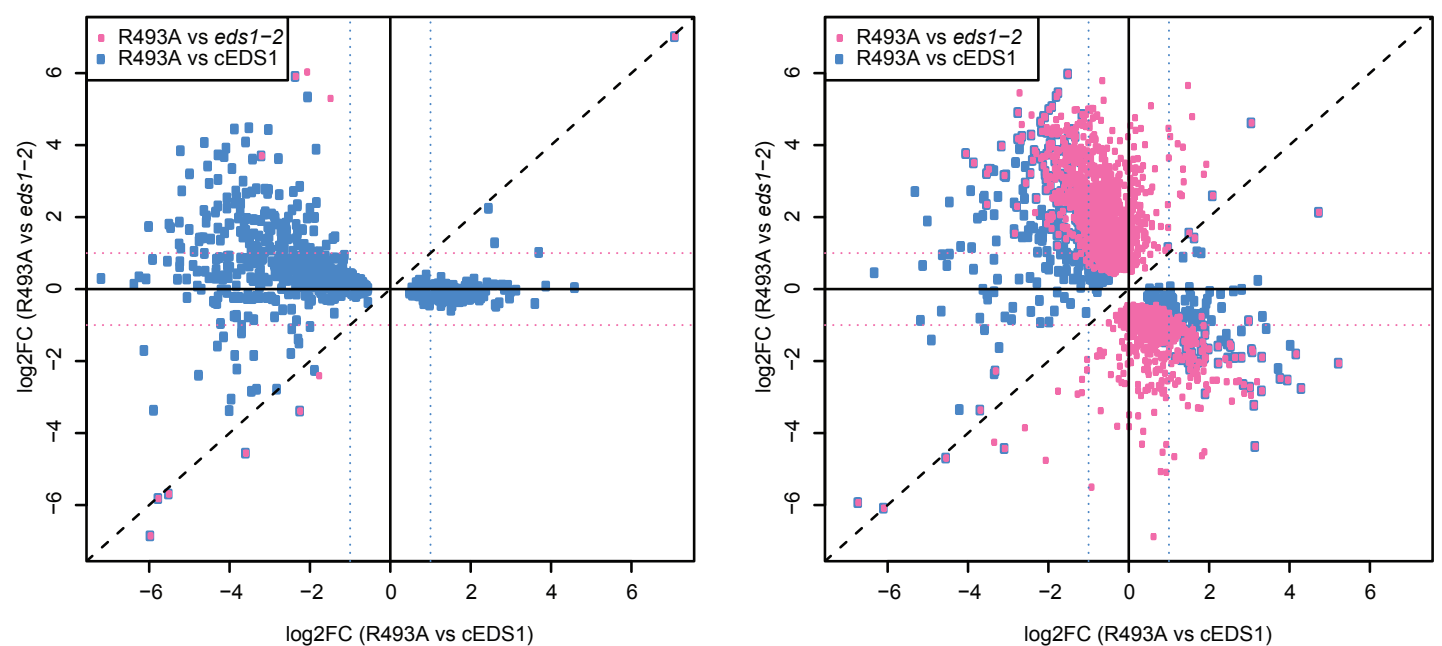

Figure 2

EDS1-R493A delays TNL transcriptional reprogramming

A. Four-week-old plants were infiltrated with Pst AvrRps 4 (OD600- 0.005) and free SA was quantified at 0, 8 and 24 hpi. Bars represent means \pm $\mathrm{SE}$ of four biological replicates. Differences between genotypes were analysed using t-test (Bonferroni corrected, $\mathrm{p}<0.05$ ). Similar results were obtained in two independent experiments. B. Four-week-old plants were infiltrated with 10mM MgCl2 (mock) or Pst AvrRps4 (OD600- 0.005), and leaf samples collected at 24 hpi. PRI transcripts were measured using qRT-PCR and normalized to GapDH. Bars represent means \pm SE of three biological replicates. Differences between genotypes were analysed using t-test (Bonferroni corrected, $\mathrm{p}<0.05$ ). C. A 2-dimensional scatter plot comparing R493A vs eds1-2 and R493A vs cEDS1 at 8 and 24 hpi with Pst AvrRps4. Plots depict differentially expressed genes (DEG) between R493A vs eds $1-2$ (pink dots) and R493A vs cEDS1 (blue dots). DEG represented have been filtered with a $|\log 2 \mathrm{FC}| \geq 1, \mathrm{FDR} \leq 0.05$. 


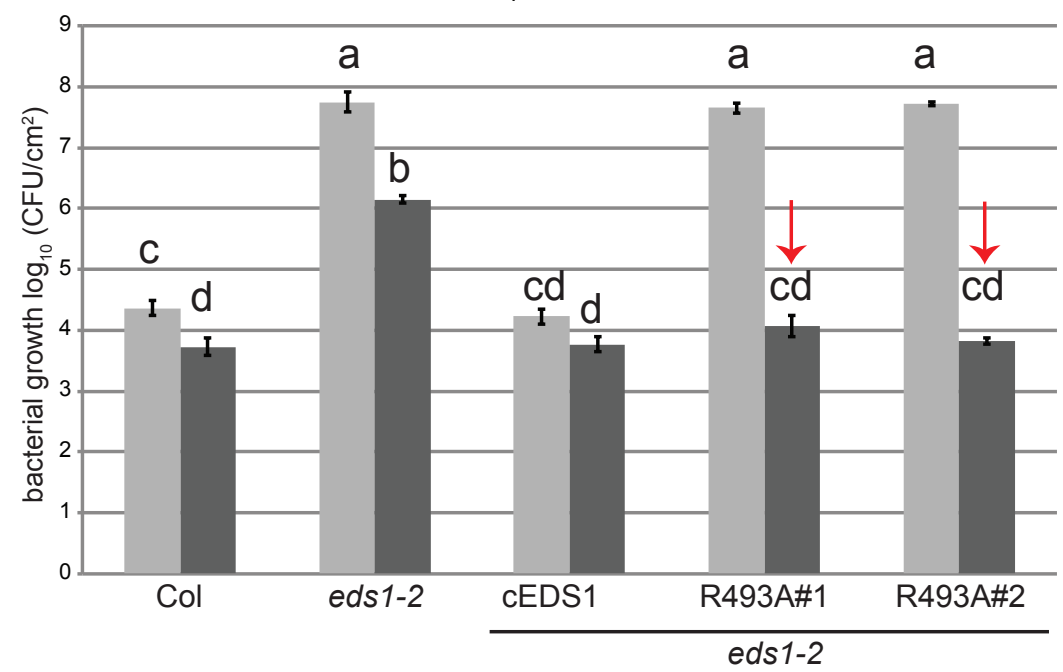

B

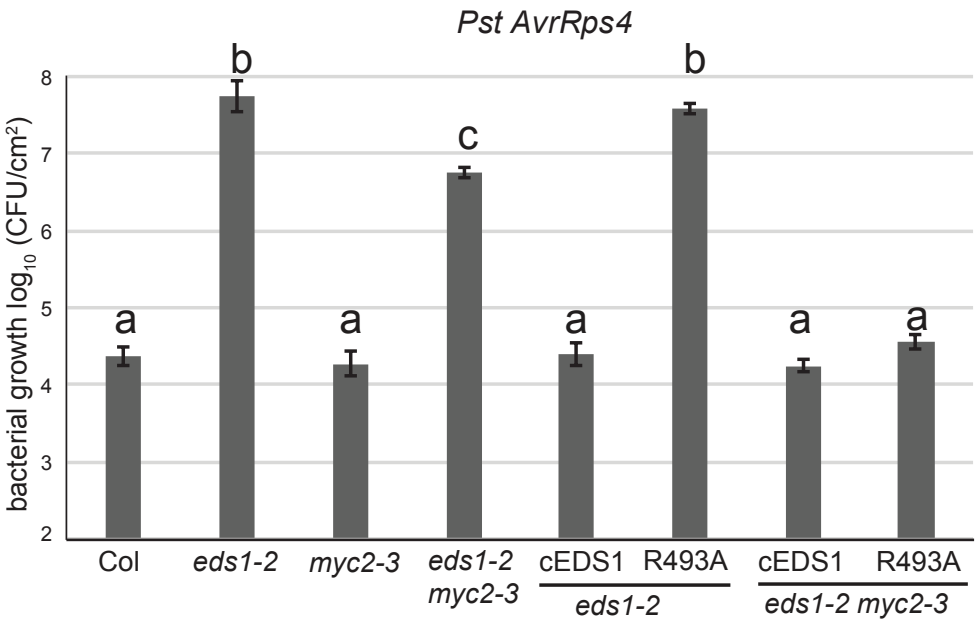

C

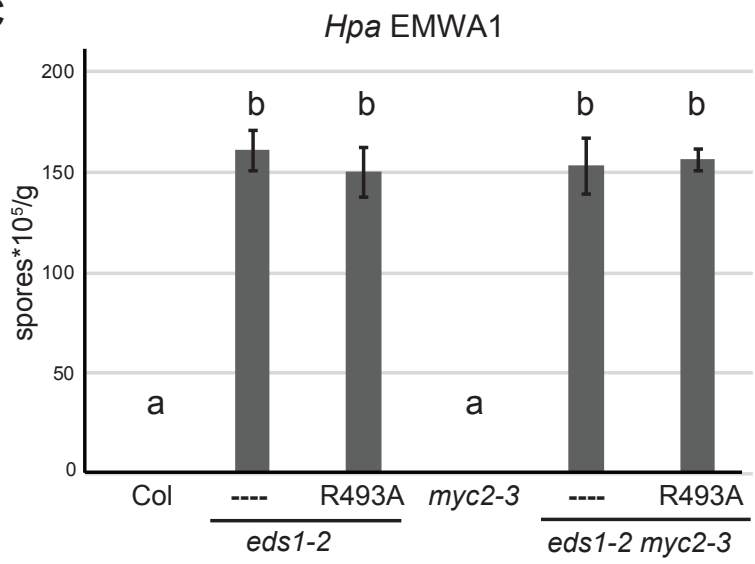

Figure 3

EDS1-R493A fails to antagonize bacterial COR-stimulated MYC2 in TNL ETI

A. Four-week-old Arabidopsis plants of the indicated genotypes were infiltrated with Pst AvrRps4 or Pst $\Delta$ Cor AvrRps4 (OD600 - 0.0005). Bacterial titers were determined at 0 and 3 dpi. No significant difference was observed between lines and treatments at 0 dpi. Bars represent mean of four biological replicates \pm SE. Differences between genotypes were analysed using ANOVA (Tukey’s HSD, $\mathrm{p}<0.005$ ). Similar results were obtained in three independent experiments. B. Four-week-old Arabidopsis plants of the indicated genotypes were infiltrated with Pst AvrRps4 (OD600 - 0.0005). Bacterial titers were determined at 0 and 3 dpi. No significant differences were observed at 0 dpi. Bars represent mean of four biological replicates \pm SE. Differences between genotypes were analysed using ANOVA (Tukey’S HSD, $p<0.005$ ). Similar results were obtained in three independent experiments. C. The indicated genotypes were infected with Hpa EMWA1 and oomycete sporulation was quantified at 5 dpi. Data from three independent experiments were combined and differences between genotypes analysed using ANOVA (Tukey’S HSD, p $<0.005$ ). 
A

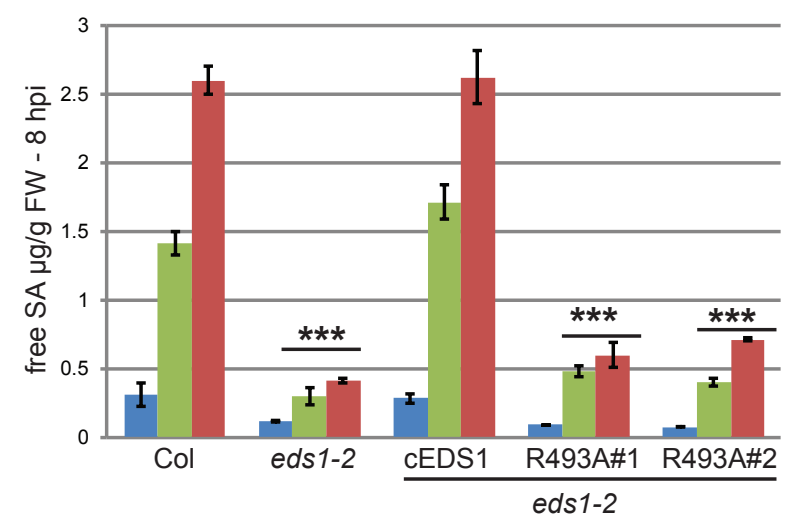

C

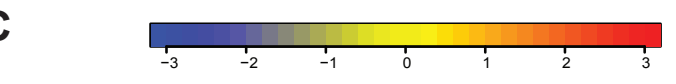

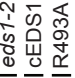

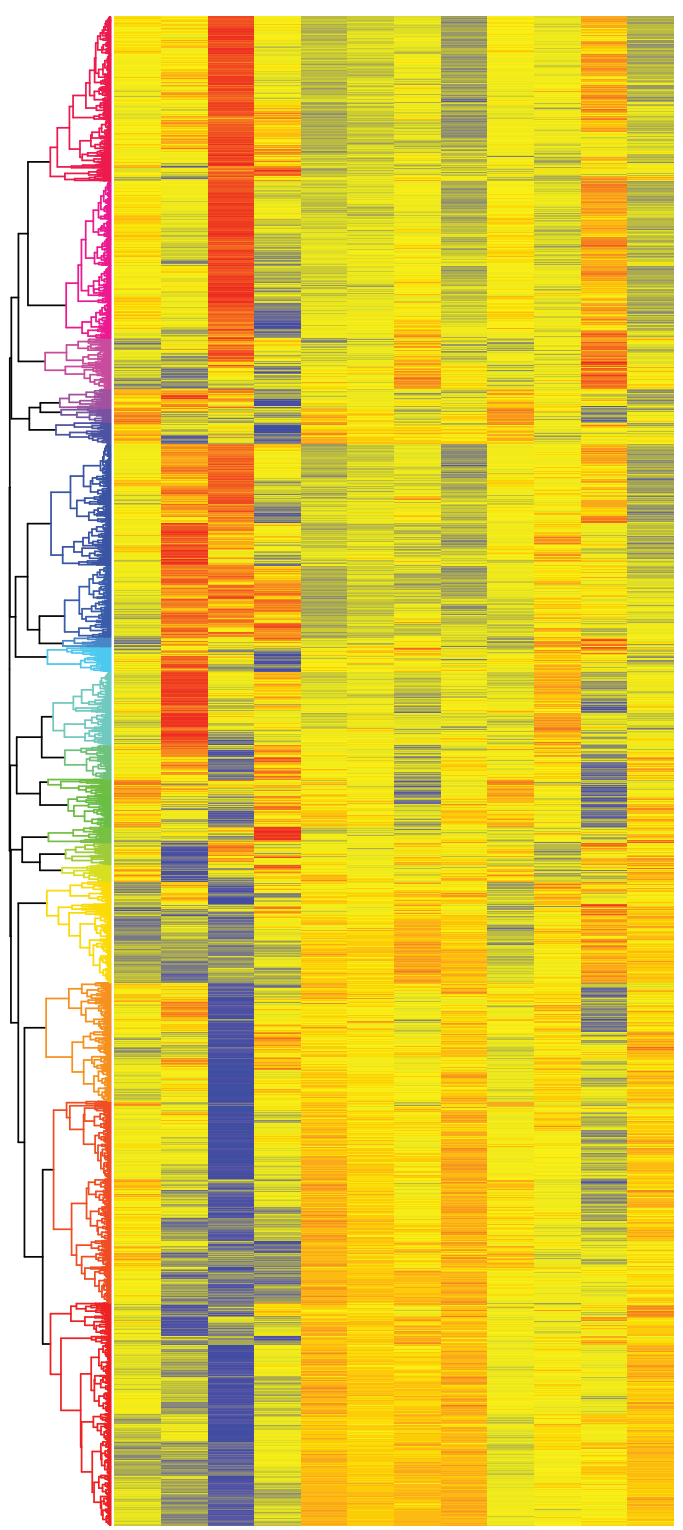
बत्रकत्र
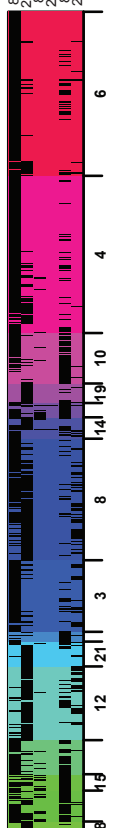

8hpi 24hpi 8hpi 24hpi 8hpi 24hpi 8hpi 24hpi 8hpi 24hpi 8hpi 24hp $\frac{\text { Avr }_{\text {eds1-2 }}}{\Delta \text { Cor }} \frac{\text { Avr }}{\text { CEDS1 }} \frac{\frac{\text { Cor }}{\text { R493A }}}{\frac{\text { Avr }}{\Delta \text { Cor }}}$
B

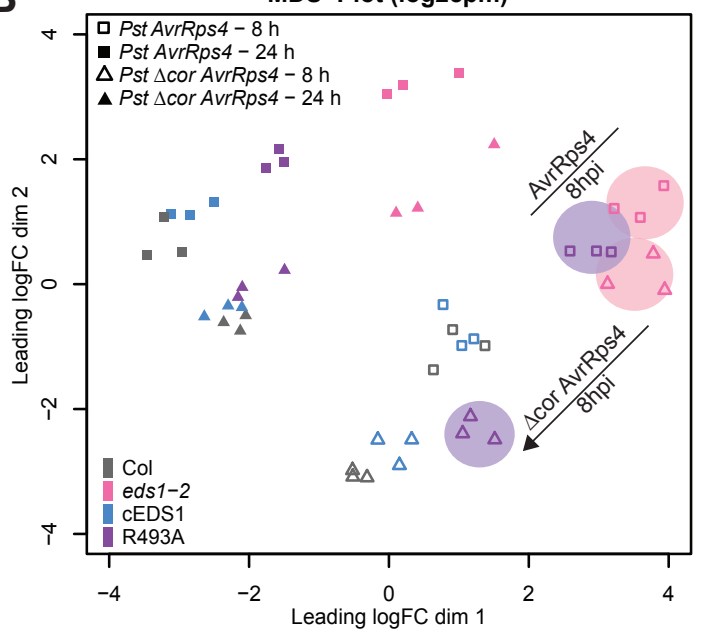

D

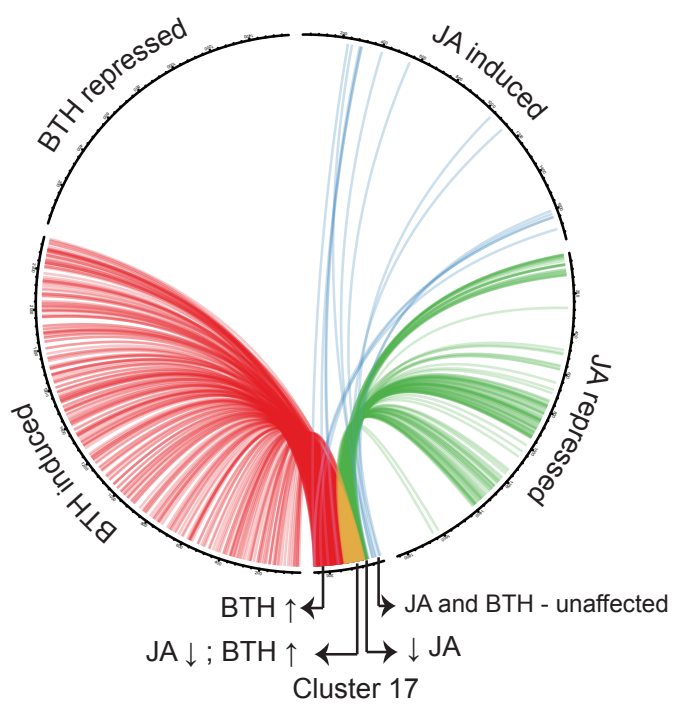

COR-antagonized NLR genes in R493A

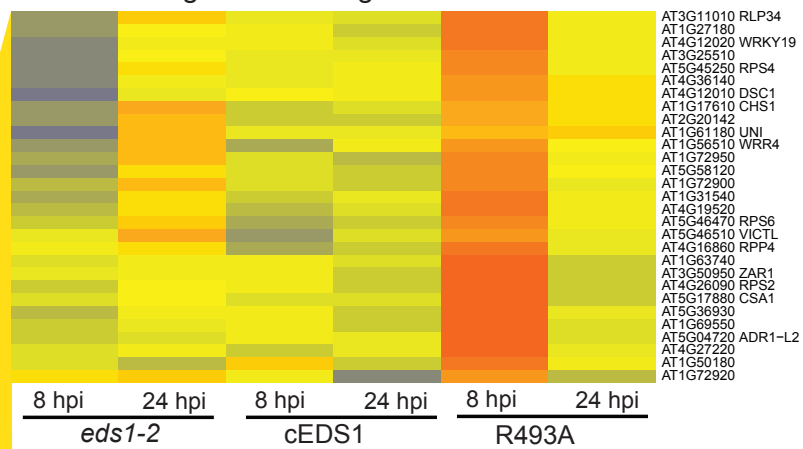

COR-antagonized WRKY genes in R493A

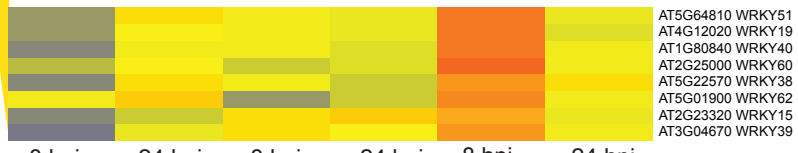

$\frac{8 \mathrm{hpi} 24 \mathrm{hpi}}{\text { eds1-2 }} \frac{8 \mathrm{hpi} 24 \mathrm{hpi}}{\mathrm{cEDS} 1} \frac{8 \mathrm{hpi} 24 \mathrm{hpi}}{\mathrm{R} 493 \mathrm{~A}}$

Figure 4

for figure legend see fig. 7 


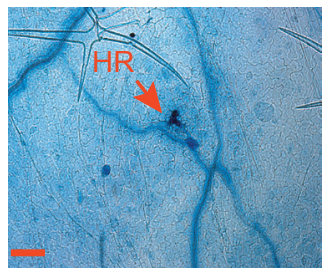

Col

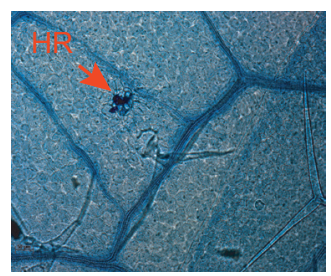

R493K\#1

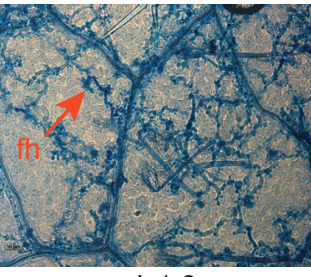

eds $1-2$

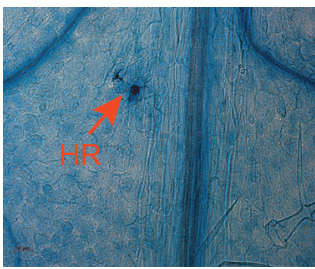

R493K\#2

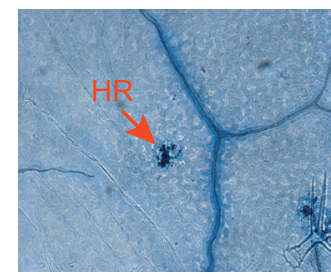

cEDS1

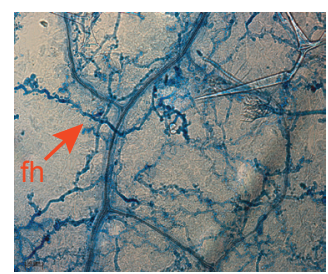

R493E\#1

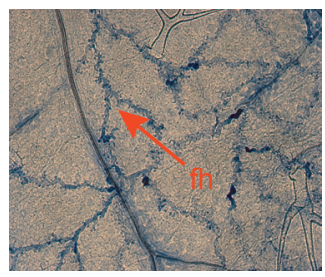

R493A

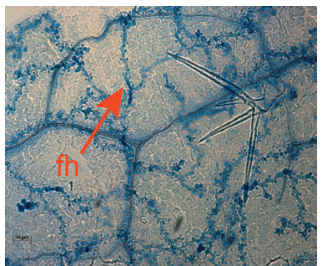

R493E\#2

eds1-2

B

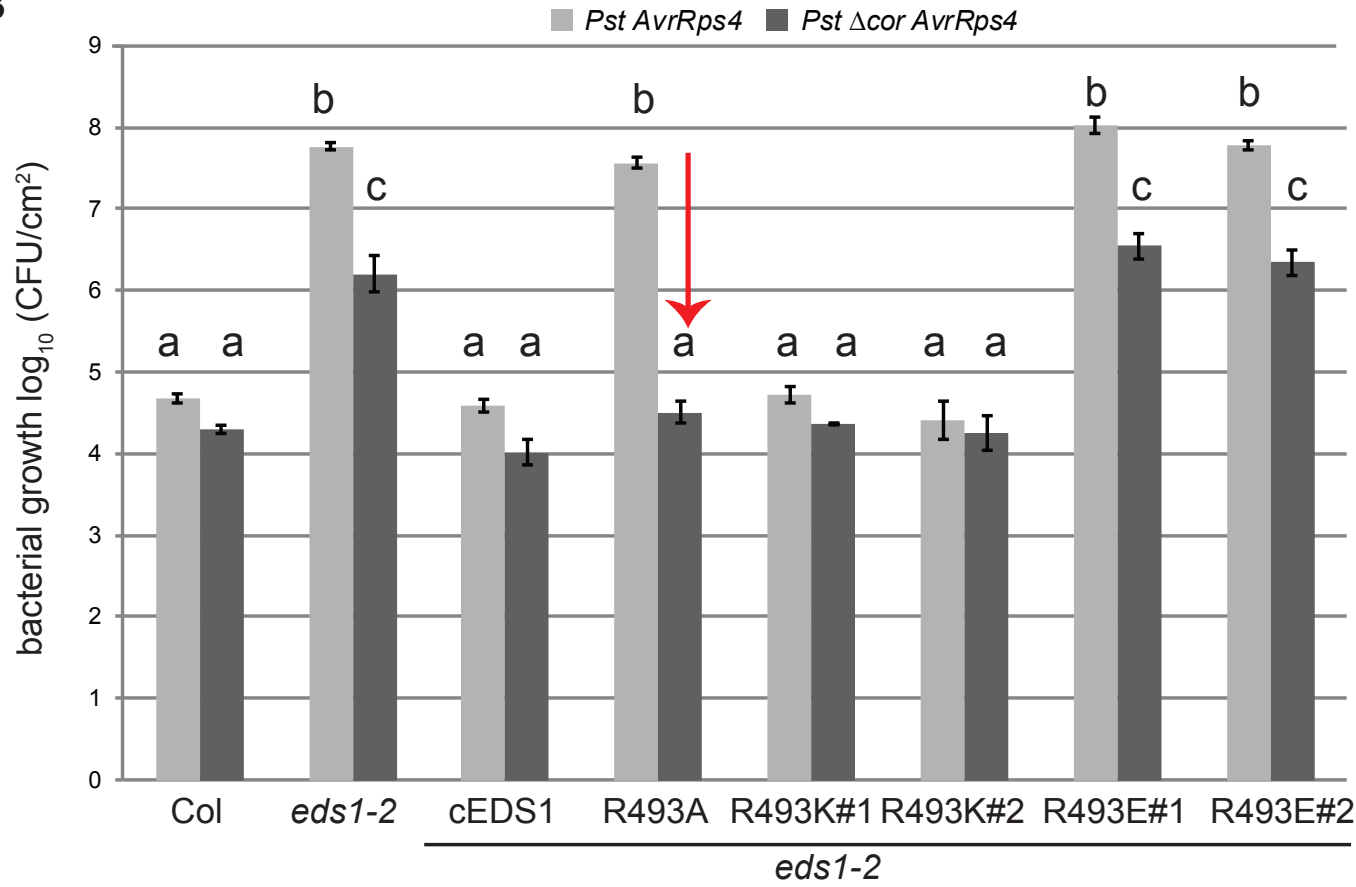

C

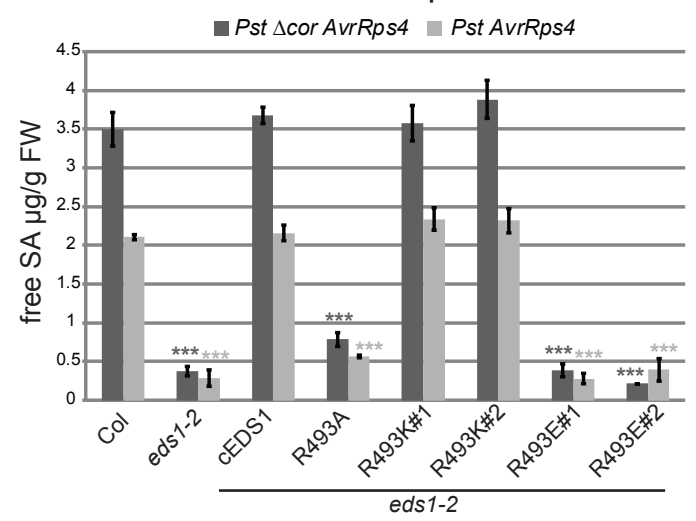

free SA - 24 hpi

- Pst $\Delta$ cor AvrRps4 Pst AvrRps4

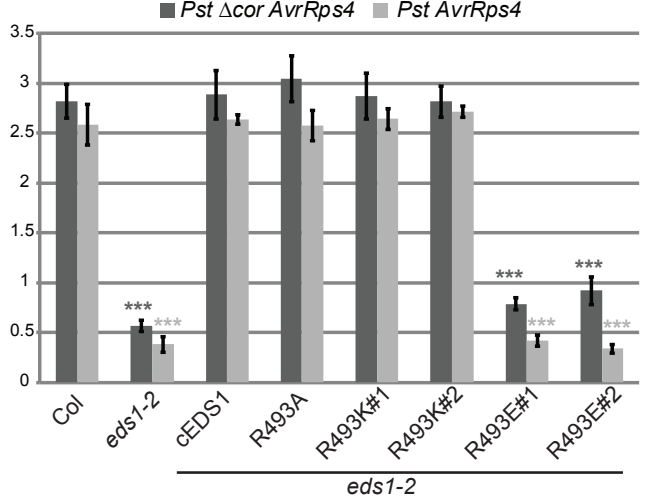

Figure 5

A positive charge at EDS1-R493 is essential for TNL immunity

A. $R P P 2$ resistance phenotypes of two-week-old control and Arabidopsis transgenic lines expressing cEDS1 and R493 mutants, as indicated. Hpa EMWA1 infected leaves were stained with trypan blue at 5 dpi. Scale bar represents $100 \mu \mathrm{m}$. Each image is representative of $>18$ leaves from two independent experiments. HR, hypersensitive response; fh, pathogen free hyphae. B - Four-week old Arabidopsis plants of the indicated genotypes were infiltrated with Pst AvrRps4 or Pst $\Delta$ cor AvrRps 4 (OD600 - 0.0005). Bacterial titers were determined at 3 dpi. Bars represent means of three biological replicates $\pm \mathrm{SE}$. Differences between genotypes were analysed using ANOVA (Tukey'S HSD, p-value $<0.05$ ). Similar results were obtained in three independent experiments. C - Four-week-old plants were infiltrated with Pst AvrRps4 or Pst $\Delta$ cor AvrRps 4 and free SA was quantified at 8 and 24 hpi. Bars represent means \pm SE of three biological replicates. Differences between genotypes within treatment were analysed using t-test (Bonferroni corrected, $* * *$ - $<<0.05$ ) relative to Col. Similar results were obtained in two independent experiments. 


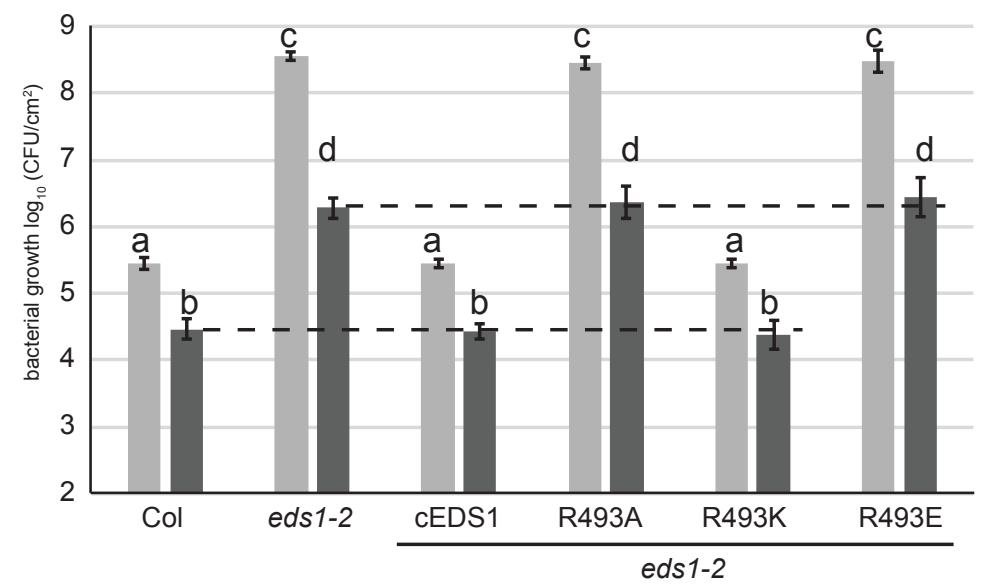

B

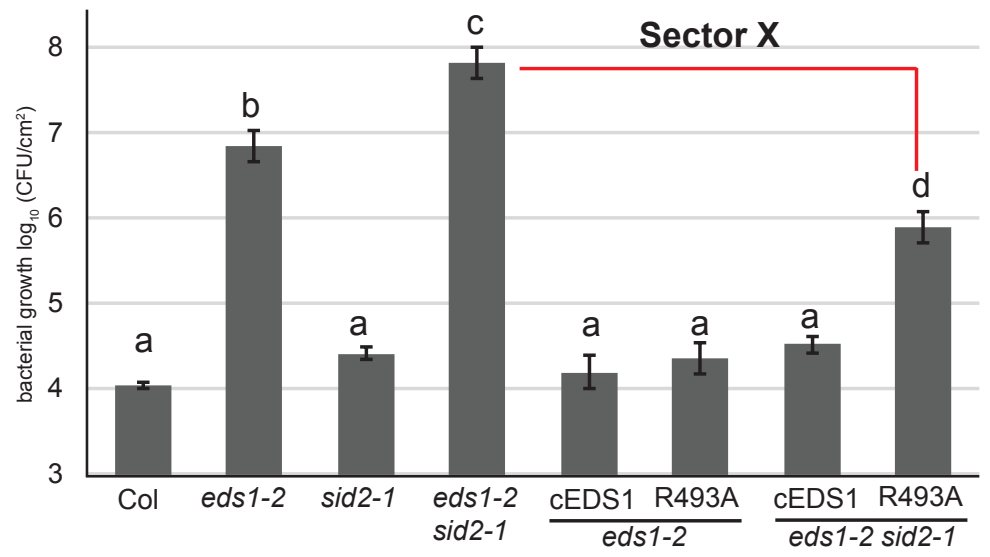

C

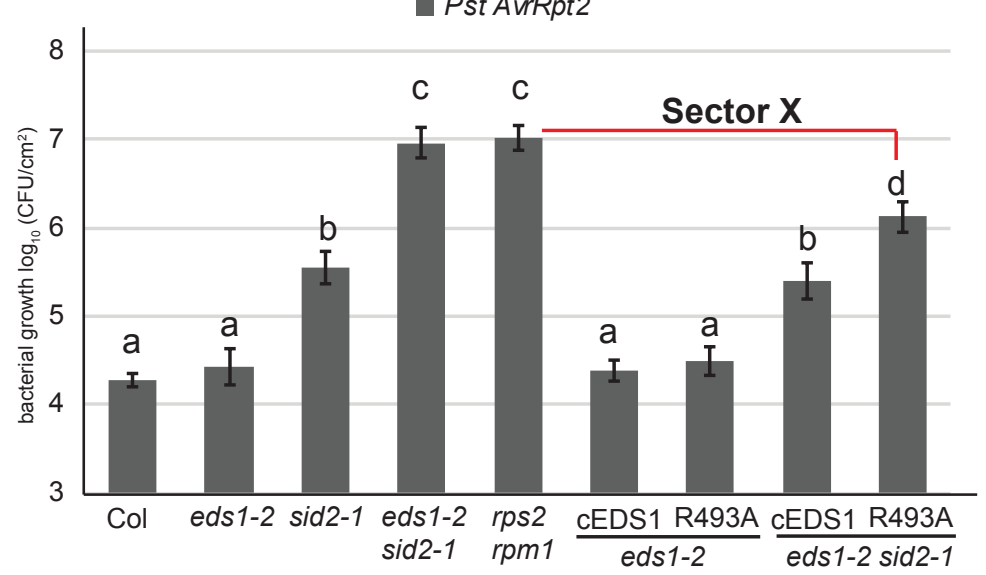

\section{Figure 6}

\section{EDS1-R493 signals in TNL and CNL (RPS2) immunity}

A. Four-week-old Arabidopsis plants of the indicated genotypes were infiltrated with Pst DC3000 or Pst $\Delta$ cor (OD600 - 0.0005). Bacterial titers were determined at 0 and $3 \mathrm{dpi}$. No significant difference was observed between lines and treatments at 0 dpi. Bars represent mean of four biological replicates \pm SE. Differences between genotypes were analysed using ANOVA (Tukey's HSD, p-value $<0.005$ ). Similar results were obtained in three independent experiments. B. Four-week old Arabidopsis plants of the indicated genotypes were infiltrated with Pst $\Delta$ cor AvrRps4 (OD600 - 0.0005). Bacterial titers were determined at 0 and 3 dpi. No significant difference was observed at 0 dpi. Bars represent mean of four biological replicates \pm SE. Differences between genotypes were analysed using ANOVA (Tukey'S HSD, $p$-value $<0.005$ ). Similar results were obtained in three independent experiments. C. Four-week-old Arabidopsis plants of the indicated genotypes were infiltrated with Pst AvrRpt2 (OD600 - 0.0005). Bacterial titers were determined at 0 and 3 dpi. No significant difference was observed at 0 dpi. Bars represent mean of four biological replicates \pm SE. Differences between genotypes were analysed using ANOVA (Tukey'S HSD, p-value $<0.005$ ). Similar results were obtained in three independent experiments. 


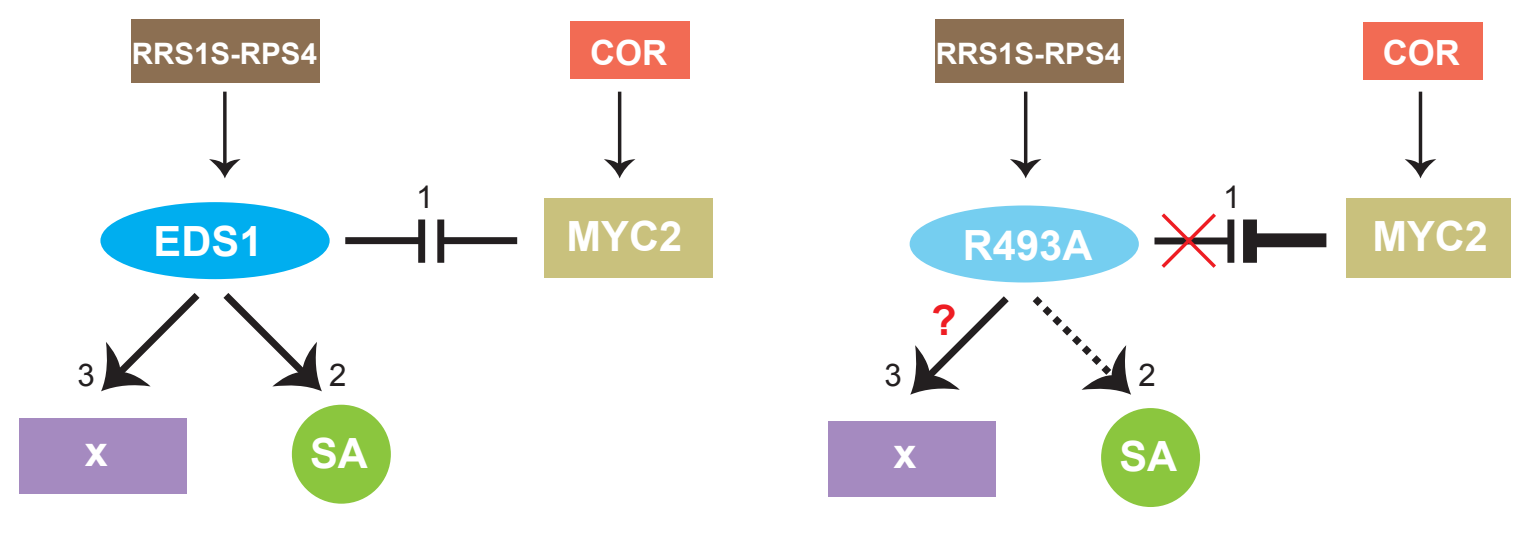

Figure 7

A model of EDS1 signalling branches in RRS1S RPS4 ETI

A three-pronged ETI signalling model derives from comparisons of wild-type EDS1, EDS1-R493A and eds 1-2 phenotypes in this study. Three interconnected EDS1 functions contribute to robust TNL ETI. 1) TNL-activated wild-type EDS1 effectively counters COR antagonism of immunity gene expression via MYC2. The defective EDS1-EP domain mutant R493A is susceptible in ETI against Pst AvrRps4 due to its inability to counter COR/MYC2 antagonism. 2) EDS1 boosts SA accumulation independently of antagonising MYC2 while EDS1-R493A delays SA accumulation (dashed lines) independently of COR repressive effects. 3) An additional EDS1 branch (X) in TNL (RRS1S RPS4) ETI is revealed after removing ICS1/SA and COR effects. The EDS1 EP-domain, and more specifically EDS1R493, is also necessary for this resistance branch. The nature of branch $\mathrm{X}$ requires further study.

\section{Figure 4}

\section{Delayed immune signalling in R493A mutant plants is independent of COR}

A. Four-week-old plants were infiltrated with $10 \mathrm{mM} \mathrm{MgCl} 2$ (mock), Pst AvrRps 4 or Pst $\Delta$ Cor AvrRps 4 and free SA was quantified at 8 hpi. Bars represent means $\pm \mathrm{SE}$ of three biological replicates. Difference between genotypes were analysed using t-test (Bonferroni corrected, $\mathrm{p}<0.05$ ). Similar results were observed in three independent experiments. B. A multi-dimension (MDS) scaling plot of differentially expressed genes showing R493A transriptional changes at 8 (open symbols) and 24 hpi (closed symbols). Encircled samples of eds 1-2 (pink) and R493A (purple) highlight transcriptional trends with and without bacterial COR in Pst AvrRps4-triggered immunity. C. A heatmap depicting DEG at 8 and 24 hpi normalized to Col $(\mathrm{p}<0.05)$ after hierarchial clustering. Samples were harvested at 8 and 24 hpi with Pst AvrRps4 (Avr) and Pst $\Delta$ Cor AvrRps 4 ( $\triangle$ Cor). Cluster \#17 contains genes that are upregulated at 8 hpi with Pst $\triangle$ Cor AvrRps 4 but not Pst AvrRps4 in R493A only. Expansion (right) highlights a subset of NLR and $W K R Y$ genes in cluster \#17 that are involved in immunity whose expression is antagonized by COR in R493A (see table S3). D. Circos plot showing overlap of 383 genes in cluster \#17 with genes regulated by JA and BTH from other studies (Hickmann et al.,2017; Yang et al.,2017). Genes differentially expressed in cluster \#17 and other datasets are marked by connecting lines. Genes repressed by JA and induced by BTH (red and green lines converging to yellow, 113); genes repressed by JA and not expressed by BTH (green lines, 19); genes induced by BTH and not expressed in JA dataset (red lines, 169). 